\title{
Relocating shared automated vehicles under parking constraints: assessing the impact of different strategies for on-street parking
}

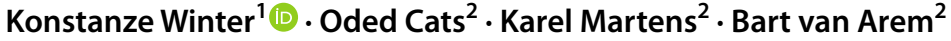

Published online: 27 May 2020

(C) The Author(s) 2020

\begin{abstract}
With shared mobility services becoming increasingly popular and vehicle automation technology advancing fast, there is an increasing interest in analysing the impacts of large-scale deployment of shared automated vehicles. In this study, a large fleet of shared automated vehicles providing private rides to passengers is introduced to an agent-based simulation model based on the city of Amsterdam, the Netherlands. The fleet is dimensioned for a sufficient service efficiency during peak-hours, meaning that in off-peak hours a substantial share of vehicles is idle, requiring vehicle relocation strategies. This study assesses the performance of zonal pro-active relocation strategies for on-demand passenger transport under constrained curbside parking capacity: (1) demand-anticipation, (2) even supply dispersion and (3) balancing between demand and supply of vehicles. The strategies are analysed in regard to service efficiency (passenger waiting times, operational efficiency), service externalities (driven mileage, parking usage) and service equity (spatial distribution of externalities and service provision). All pro-active relocation strategies are outperformed by a naïve remain-at-drop off-location strategy in a scenario where curbside parking capacity is in abundance. The demand-anticipation heuristic leads to the highest average waiting times due to vehicle bunching at demand-hotspots which results in an uneven usage of parking facilities. The most favourable results in regard to service efficiency and equity are achieved with the heuristics balancing demand and supply, at the costs of higher driven mileage due to the relocation of idle vehicles. These results open up opportunities for municipalities to accompany the introduction of large fleets of shared automated vehicles with suitable curbside management strategies that mitigate undesired effects.
\end{abstract}

Keywords Shared automated vehicles · On-demand transport - Vehicle relocation · Curbside parking $\cdot$ Agent-based simulation

Konstanze Winter

m.k.e.winter@tudelft.nl

1 Technion - Israel Institute of Technology, Haifa, Israel

2 Delft University of Technology, Delft, The Netherlands 


\section{Introduction}

The development of technology for automatically driven vehicles is progressing fast. This raises not only questions about the impact of fully automated vehicles (AV) on future mobility and traffic patterns, but also on their impact on the existing infrastructure. High degrees of vehicle automation allow the introduction of vehicles that drive autonomously, which can thus be shuffled from one place to another without having a human on-board. This opens up new opportunities in the field of car-sharing, in which currently one of the main challenges is to balance the supply of car-sharing vehicles with the demand for them. In this paper, we analyse the performance of a large fleet of shared automated vehicles (SAV). Such cooperative fleets bring new challenges to the operators and regulators of such mobility services, as they neither follow fixed schedules nor fixed routes. One of these challenges is the question, how to deal with idle vehicles whose services are currently not required. This is especially a pressing issue in off-peak hours, when larger number of idle vehicles need to be managed. The focus of this study is put, in particular, on strategies for relocating idle SAV, and how these influence the performance of the transport service offered by SAV, as well as the consumption of parking space and the overall mileage driven by the SAV. The constraints caused by the scarcity of parking space is an issue often overlooked in past studies simulating the operation of SAV or similar on-demand transport services with unlimited parking facilities, despite the substantial impact such constraints have on the performance of such a service.

The SAV transport service in this paper is envisioned as an on-demand transport service operated by a fleet of automated vehicles that require no human intervention (level 5 automation; or level 4 automation if only operated on a selection of suitable roads). In countries with high labour cost, on-demand systems are currently either highly subsidized (and often limited to users with special needs or those living in remote areas) or expensive (and primarily used by strong socio-economic groups), with operating costs often more than three times higher than for schedule-based transit services (Anderson et al. 2014; Wright et al. 2014). By deploying AV, flexible door-to-door services could be implemented on a larger scale at much lower costs, which could become an important enrichment of the current schedule-bound public transit services. Another advantage of SAV is, that-differing to on-demand transport services operated by human drivers (e.g. ride-sourcing)—vehicles can be programmed to fully comply with the central dispatcher's orders and can relocate themselves accordingly (Zhang et al. 2016), and that vehicles belonging to the same fleet are not competing against each other for revenue. The results of the model analysis in this study would also hold for any non-automated on-demand transport service strictly following the advice of the central dispatcher.

For an on-demand system operated by SAV to have sufficient spatial coverage and level-of-service, large fleets of AV have to be employed, as various simulation studies have shown (Alonso-mora et al. 2017; Bischoff and Maciejewski 2016a; Chen et al. 2016; International Transport Forum 2015). In these studies, thousands of shared AVs have been introduced to serve demand in large cities. Their search for the appropriate fleet sizes is mainly driven by targets concerning the level of service, most commonly expressed in passengers' waiting times and/or trip times, either as an average or in terms of a minimum level of service. By setting these kinds of boundary conditions, fleet sizes are dimensioned to cater for the maximum demand occurring during peak hours. Consequently, there will be idle vehicles during off-peak hours, which can either be "stored" on the road network by letting the vehicles cruise empty or park on on-street parking facilities, or be sent to 
off-street parking facilities (depots). The problem of the relocation of idle vehicles in the operation of large fleets of vehicles is one of the central challenges and a potential barrier for the introduction of large-scale shared on-demand transport services, be it for conventional taxi services or services operated by SAV (Babicheva et al. 2018; Dandl and Bogenberger 2019; Sayarshad and Chow 2017; Winter et al. 2017).

In this paper, idle vehicle relocation is not regarded solely as a supporting step to an efficient vehicle dispatching, but also as a means to manage idle vehicles not in use according to principles reflecting all stakeholders' interests. With this vision on vehicle relocation, we move from the question on where to simply "store" idle vehicles on to the question, how vehicle relocation can effectively improve the service operation of a fleet of vehicles while mitigating undesired external effects caused by the service. In particular, the performance of three heuristics for the proactive relocation strategies Demand Anticipation, Supply Anticipation and Demand-Supply Balancing is tested. These relocation strategies are compared in three aspects for selected key-performance indicators: (1) performance of the SAV system, (2) external effects and (3) service equity provided by the SAV system. The relocation strategies are simulated for this analysis in an agent-based simulation model of a large-scale case study based on the city of Amsterdam, the Netherlands. The main contributions of this study can be summarized by the following:

- Comparison of three pro-active relocation heuristics for shared automated vehicles under parking constraints.

- Introducing a fleet of shared automated vehicles into an agent-based model for a largescale case study based on the city of Amsterdam.

- Holistic impact analysis of SAV in regard to service efficiency, service provision equity, and service externalities.

The remaining of this paper is structured as follows: In "Relocating shared automated vehicles" section, the problem of vehicle relocation for shared automated vehicles is described in more detail and approaches to this problem as described in the literature are discussed, and the three relocation strategies tested in this study are defined. In "Case study application" section, the modelling environment, the description of the network, the demand and the supply for SAV are described. In "Results" section, the simulation results are presented and analysed according to the impact criteria stated above. The paper is concluded with "Discussion and conclusion", which provides a discussion of the results and an outlook on future research.

\section{Relocating shared automated vehicles}

\section{Problem description}

In Fig. 1, a schematic overview is given of the chain of operations necessary for the deployment of SAV: vehicle dispatching, vehicle routing, and vehicle relocating. Vehicle routing and vehicle dispatching are integral steps of the operation of SAV. Vehicle relocation, however, is an optional step, as it can be alternatively decided to only move the vehicle from its latest passenger drop-off location to the next passenger pick-up location once the vehicle has been dispatched to a new request (which can occur instantly in case there is a queue of unserved requests). However, adding the additional step of 


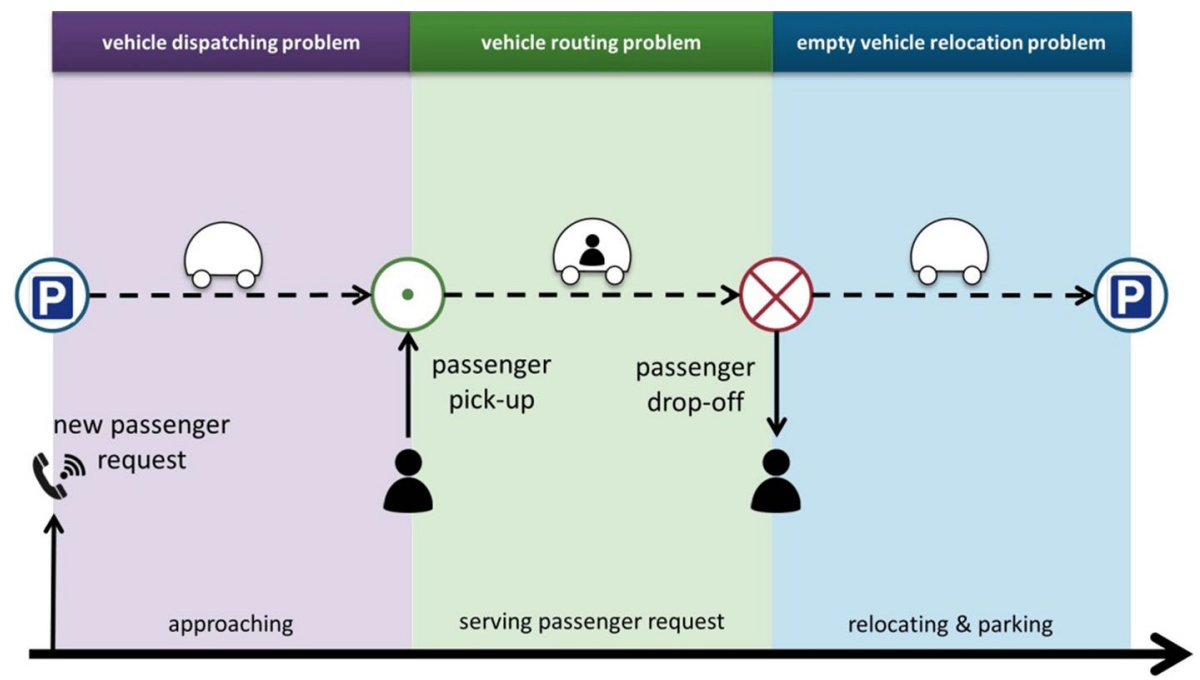

Fig. 1 Chain of problems for operating an on-demand transport service: dispatching, routing and relocating

relocating the vehicle to a strategically chosen parking location can potentially improve the overall performance and level-of-service. Furthermore, it would also be essential for operating real-world SAV systems due to limited parking facilities in urban environments. This is especially true in times where there is little demand for the service, e.g. during off-peak hours, which results in an oversupply of vehicles.

The relocation of idle vehicles has been described by the Empty Vehicle Redistribution Problem, which falls in the category of Vehicle Routing Problems, a subcategory of the Traveling Salesman Problem (Babicheva et al. 2018). Idle vehicle relocation has also been described as the Idle Vehicle Propositioning Problem, as a subcategory of Facility Location Problems (Sayarshad and Chow 2017). These problems are NP-hard and are notoriously difficult to solve, especially in dynamic settings. For this reason, applying heuristics for the dispatching and relocation of vehicles in large cooperative fleets is the most common approach in simulation studies of large-scale on-demand transport systems.

\section{Network}

A directed graph $G(V, E)$ is used for representing the dynamic transport service network with $E$ being a set of arcs (edges) and $V$ being a set of vertices. Each vertex $v$ represents an intersection between arcs and each arc $e$ is described by its characteristics: link length, the maximum allowed driving speed, free flow capacity and the maximum parking capacity denoted by $\kappa_{e}^{\max }$. At a discrete moment in time $\tau$, the time-dependent variables of current driving speed and the current free parking capacity $c_{e}(\tau)$ describe the state of the arc. Furthermore, $Z$ denotes a set of zones, described by the set of arcs present in that zone $E_{z}$ and the time-dependent variables of the current free parking capacity of all arcs in zone $z, c_{z}(\tau)=\sum_{e \in E_{z}} c_{e}(\tau)$. 


\section{Demand for SAV}

The demand for SAV has been modelled based on the general population dynamics implemented in MATSim (see Nagel et al. 2016). In MATSim, travellers follow a daily plan, which consists of a set of activities that they want to perform. For each activity, the location is known, as well as the desired start and end times and the mode the agents intend to use to reach the activity. Each traveller memorizes a set of these plans, for which the plans can vary in activity start and end times, modal choices or route choices, but always show the same sequence of activities. In the course of a repeated simulation of the same day, agents can try out different plans and improve parts of the plans according to predefined behavioural strategies also known as 'innovation rules'. The plan selection is based on the concept of utility maximisation, as performing an activity and travelling towards an activity are scored based on their perceived utility. The repeated plan innovation and plan selection in the face of the resulting traffic states leads to an optimization of the agents' plans through the co-evolutionary search for the resulting equilibrium (Balmer and Rieser 2009), which is de facto also leading to a user equilibrium on the road network.

The demand for SAV is expressed in the form of individual requests. Each individual request $q \in Q$ is launched by an agent at time step $\tau$ at a pick-up (origin) location on an $\operatorname{arc} e_{q}^{o}$, where $Q$ is the set of all travel requests for SAV rides in the network under consideration. Information concerning the downstream drop-off location of a request is not used during the vehicle dispatching and relocating process. All requests that are not yet dispatched or are in the process of being dispatched are stored in the time-dependent set of open requests $Q(\tau)$.

\section{Supply of SAV}

Each SAV follows, similarly to the travellers, a schedule for the whole day, which is imposed on it by a central dispatcher. In contrast to the travellers, who update their plans from day to day, the vehicles' schedules are updated within each simulated day in response to passengers' requests.

The SAV are stored in a set of vehicles $K$. Each vehicle $k \in K$ is described by its length, the maximum vehicle speed, its current location denoted by $e_{k}(\tau)$ and its current dispatching status. Vehicles are grouped in subsets according to their dispatching status: the subset $K^{\text {serve }}(\tau)$, in which all vehicles currently assigned to dispatch a request (and are therefore moving-either empty towards a pick-up point or with a passenger on-board heading towards the drop-off location) are stored, and the subset $K^{\text {idle }}(\tau)$, in which all idle vehicles currently not assigned to dispatch a request are stored. The latter has a subdivision, the set of vehicles that are not in use and relocating according to one of the relocating strategies $K^{\text {reloc }}(\tau)$ and the vehicles that are idle and parked $K^{\text {park }}(\tau)$. Relocating vehicles are, despite being on the move, considered to be idle and can at any moment be diverted from their relocation path in order to serve an incoming request. It holds that $K^{\text {serve }}(\tau) \cup K^{\text {idle }}(\tau)=K$ and that $K^{\text {reloc }}(\tau) \cup K^{\text {park }}(\tau)=K^{\text {idle }}(\tau)$ since these sets are mutually exclusive and collectively exhaustive. 


\section{Vehicle relocation heuristics}

In regard to the transport service provided by the SAV envisioned in this study, many parallels can be drawn between SAV and the current taxis, which also provide ondemand transport services. For this reason, we include in the review of relocation strategies for SAV also the strategies applied to taxis today. There are multiple heuristic vehicle relocation strategies for vehicles of on-demand services, which can be divided into two groups: reactive and proactive relocation strategies (Babicheva et al. 2018). Reactive relocation means that vehicles relocate only upon passenger request, while proactive relocation strategies relocate vehicles in anticipation of future demand and/ or supply states. In the latter case, the step of relocating and dispatching are interconnected. The different strategies differ also in regard to their overall goal: while some aim at increasing the chance for an individual vehicle to be dispatched to requests as often as possible, others are designed to improve the overall service of a fleet, to reduce undesired externalities or to support scheduled public transport services in regions with underdeveloped coverage, e.g. when used as last-mile service.

Two reactive relocations strategies can be distinguished: parking and cruising. Reactive strategies applying "parking" either park idle vehicles at their last drop-off location, which in the following we refer to as the strategy Remain, or send them to a taxi stand or depot. Though the reactive relocation strategy of parking at the last drop-off location is not commonly observed in the operation of demand-responsive transport services, it is often selected as a default option in simulation studies featuring SAV or similar on-demand transport services (Bailey and Clark 1992; Ben-Dor et al. 2019; Fagnant and Kockelman 2014; Maciejewski et al. 2016; Winter et al. 2017), implicating that idle vehicles park at the last drop-off location regardless of parking (capacity) constraints. The relocation strategy of Cruising is a phenomenon that can be observed in the real world when drivers of on-demand transport services are searching for potential customers while avoiding parking search and possible parking fees, as is the case for regular taxis, ride-hailing services and many para-transit services in the Global South (Anderson 2014). Idle cruising increases the driven vehicle mileage and, by this, can contribute to congestion effects, increased fuel consumption or energy usage and increased emissions. This strategy has been included in simulation studies (Zhang et al. 2016), mainly as a means for benchmarking proposed proactive relocation strategies in regard to driven mileage and service efficiency.

In this study, vehicles are assigned to incoming requests according to the "rule-based" dispatching strategy described in (Maciejewski et al. 2016; Maciejewski and Bischoff 2015). In times of oversupply of vehicles, this dispatching strategy assigns the nearest vehicle to customer requests in a first-in-first-out (FIFO) order of the requests, and in times of undersupply of vehicles assigns the next idle vehicle to the closest open customer request. In case no open requests remain to be dispatched, the vehicles stay idle at the drop-off location of the last request they have been serving, the relocation strategy applied by default in the "rule-based" dispatching strategy is thus Remain. The Remain strategy does not take into account that in the real world, parking space and road space are limited resources. Letting vehicles simply wait at their latest drop-off location is hence an unrealistic representation of the operation of SAV or other comparable mobility services. We therefore consider three more advanced relocation strategies taking parking constraints into account, which we referred to as (1) Demand Anticipation, (2) Supply Anticipation and (3) Demand-Supply Balancing. A detailed description of these strategies as used in this study and as found in the literature is provided in the following sections. The three strategies used in this study 
if $k \in K^{\text {idle }}(\tau) \wedge k \in K^{\text {serve }}(\tau-1) \wedge Q(\tau)=\emptyset$,

//for the search of potential zones of destination, only include those with free parking available $\mathrm{Z}_{\mathrm{p}}=\left\{\mathrm{z} \in \mathrm{Z} \mid \mathrm{c}_{\mathrm{z}}(\tau)>0\right\}$

Case "Demand Anticipation":

// determine set of potential zones of destination

if $\max _{\mathrm{z} \in \mathrm{Z}}\left|\mathrm{Q}_{\mathrm{z}}\left[\tau, \tau_{\mathrm{h}}+\alpha \mathrm{h}\right]\right|>0$

for $\mathrm{i}=1$ to $\zeta$

$\mathrm{z}_{\mathrm{i}}=\underset{\mathrm{z} \in \mathbf{Z}_{\mathbf{p}}}{\operatorname{argmax}}\left(\left|\mathrm{Q}_{\mathbf{z}}\left[\tau, \tau_{\mathrm{h}}+\alpha \mathrm{h}\right]\right|\right)$

$\mathrm{Z}^{\prime} \cup\left\{\mathrm{z}_{\mathrm{i}}\right\}$

Otherwise

$$
\mathrm{z}^{*}=\underset{\mathrm{z} \in \mathbf{Z}^{\prime}}{\operatorname{argmin}}\left(\mathrm{d}\left(\mathrm{e}_{\mathrm{k}}(\tau), \mathrm{e}_{\mathrm{z}}\right)\right)
$$

$$
\mathrm{Z}_{\mathrm{p}}:=\mathrm{Z}_{\mathrm{p}} \backslash \mathrm{Z}_{\mathrm{i}}
$$

// determine closest zone with free parking available $\mathrm{z}^{*}=\underset{\mathrm{z} \in \mathrm{Z}_{\mathrm{p}}}{\operatorname{argmin}}\left(\mathrm{d}\left(\mathrm{e}_{\mathrm{k}}(\tau), \mathrm{e}_{\mathrm{z}}\right)\right)$

\section{Case "Supply Anticipation":}

$$
\begin{gathered}
\text { if } \max _{\mathrm{z} \in \mathrm{Z}}\left|K_{\mathrm{z}}^{\text {park }}\left[\tau, \tau_{h}+\alpha h\right]\right|>0 \\
\text { for } \mathrm{i}=1 \text { to } \zeta \\
\mathrm{Z}^{\prime} \cup\left\{\mathrm{z}_{\mathrm{i}}\right\} \\
\mathrm{Z}_{\mathrm{p}}:=\mathrm{Z}_{\mathrm{p}} \backslash \mathrm{Z}_{\mathrm{i}} \\
\mathrm{z}^{*}=\underset{\mathrm{z} \in \mathbf{Z}^{\prime}}{\operatorname{argmin}}\left(\mathrm{d}\left(\mathrm{e}_{\mathrm{k}}(\tau), \mathrm{e}_{\mathrm{z}}\right)\right)
\end{gathered}
$$

Otherwise

// determine closest zone with free parking available

$$
\mathrm{z}^{*}=\underset{\mathrm{z} \in \mathrm{Z}_{\mathrm{p}}}{\operatorname{argmin}}\left(\mathrm{d}\left(\mathrm{e}_{\mathrm{k}}(\tau), \mathrm{e}_{\mathrm{z}}\right)\right)
$$

Case "Demand-Supply Balancing":

$$
\begin{aligned}
& \text { if } \max _{z \in Z}\left(\left|Q_{z}\left[\tau, \tau_{h}+\alpha h\right]\right|-\left|K_{z}^{\text {park }}\left[\tau, \tau_{h}+\alpha h\right]\right|\right)>0 \\
& \text { for } \mathrm{i}=1 \text { to } \zeta \\
& \quad \mathrm{z}_{\mathrm{i}}=\underset{\mathrm{z} \in \mathrm{Z}_{\mathrm{p}}}{\operatorname{argmax}}\left(\left|Q_{\mathrm{z}}\left[\tau, \tau_{h}+\alpha h\right]\right|-\left|K_{\mathrm{z}}^{\text {park }}\left[\tau, \tau_{h}+\alpha h\right]\right|\right) \\
& \quad \mathrm{Z}^{\prime} \cup\left\{\mathrm{Z}_{\mathrm{i}}\right\} \\
& \quad \mathrm{Z}_{\mathrm{p}}:=\mathrm{Z}_{\mathrm{p}} \backslash \mathrm{Z}_{\mathrm{i}} \\
& \mathrm{z}^{*}=\underset{\mathrm{z} \in \mathbf{Z}^{\prime}}{\operatorname{argmin}}\left(\mathrm{d}\left(\mathrm{e}_{\mathrm{k}}(\tau), \mathrm{e}_{\mathrm{z}}\right)\right)
\end{aligned}
$$

Otherwise

$$
\begin{aligned}
& / / \text { determine closest zone with free parking available } \\
& \mathrm{z}^{*}=\underset{\mathrm{z} \in \mathrm{Z}_{\mathrm{p}}}{\operatorname{argmin}}\left(\mathrm{d}\left(\mathrm{e}_{\mathrm{k}}(\tau), \mathrm{e}_{\mathrm{z}}\right)\right)
\end{aligned}
$$

// determine arc on which relocating vehicle will park

$\widetilde{\mathrm{e}_{\mathrm{k}}}=\underset{\mathrm{e} \in \mathrm{E}_{\mathrm{z}^{*}}}{\operatorname{argmax}}\left(\left|\mathrm{c}_{\mathrm{e}}\left(\tau_{\mathrm{h}}+\alpha \mathrm{h}\right)\right|\right)$

Fig. 2 Pseudo-code for the relocation strategies simulated in this study

are composed of simple heuristic building blocks (described in pseudo-code in Fig. 2), making them comparable and traceable. The first strategy aims at placing idle vehicle close to future demand, the second strategy aims at distributing idle vehicles throughout the network and the third strategy aims at meeting both goals of the previous strategies by mitigating future demand-supply deficits. All strategies are put into action on a zonal level.

The relocation of an idle vehicle $k$ is performed in all cases when there is no pending unassigned request and the vehicle in question has been serving a passenger request in the previous time step and is currently idle. The relocation strategy determines the vehicle 
destination link so that it moves from its current location $e_{k}(\tau)$ to the selected destination $\operatorname{arc} e_{k}^{d}$. The three pro-active relocation strategies analysed in this paper are based on predictions of future demand and supply per zone, for which a rolling horizon time $\alpha h$ is defined, where $\alpha$ is a parameter that sets the number of horizon windows considered, each of which is $h$ minutes long. For reasons of simplicity, we make usage of our full knowledge about future requests, the expected future demand is thus the true demand based on the agents' plans, and not an estimation thereof. The results for this strategy, therefore, are an overestimation of the performance of this relocation strategy, which in reality will be subject to prediction errors.

\section{Relocation strategy "Demand Anticipation"}

Demand-anticipatory strategies relocate vehicles to places where high demand for their services is expected in the near future. They can be observed in the real world where ondemand services are not strictly regulated and drivers have to compete for customers. As drivers typically lack information on (future) demand, this can lead to many vehicles heading to the same high demand locations, causing an imbalance in the supply of vehicles, which can lead to overall lower system performance, lower service availability in some areas, undesired bunching of vehicles in the network and an increase in driven mileage (Anderson 2014; Cetin and Deakin 2019; Sayarshad and Chow 2017; Zheng et al. 2018). The simulation of demand anticipatory strategies are either based on the assumption of full knowledge of the future demand (Hörl et al. 2019; Winter et al. 2017; Zhang et al. 2016), or at least of the expected arrival rates (Sayarshad and Chow 2017; Zhang et al. 2016), or aim at modelling the risk-taking preferences of the operator (van Engelen et al. 2018). The quality of the forecast of demand for SAV depends on the aggregation level of the spatiotemporal demand forecast. A framework for tackling this problem is presented in Dandl et al. (2019). They show that using more aggregated zoning (edge length of $2.5 \mathrm{~km}$ ) for the demand forecast reduces empty mileage and hence improves the service provided by SAV.

For the strategy Demand Anticipation, as formulated in this study, expected future requests are determined per zone for the time span between $\tau$ and $\tau+\alpha h$, and are stored in the temporary set of cumulative open requests $Q[\tau, \tau+\alpha h]$. From the set of $\zeta$ zones with the largest amount of open request, the zone $z^{*}$ closest to the current position of the vehicle $e_{k}(\tau)$ is chosen, under the condition that at there is currently at least one free parking spot available in that zone (see Fig. 2). The distance $d$ to the zone is measured from the current location of the vehicle $e_{k}(\tau)$ and the arc closest to the centre of a zone, $e_{z}$. Within the selected zone, the arc with the largest number of free parking spots at the time step $\tau+\alpha h$ in that zone is selected to be $\tilde{e}_{k}$. The vehicle will relocate to the pick-up location of that request and park there upon arrival. To ensure that this will be possible, a parking spot is reserved on $\operatorname{arc} e_{q}^{o}$ at the time of the selection of the location, to which the vehicle will relocate to.

In case that the pick-up location of none of the requests in $Q\left[\tau, \tau_{h}+\alpha h\right]$ is in a zone with a residual parking capacity, the vehicle will park in the zone closest to the vehicle with free parking. Again, the arc with the largest number of free parking spots at the current time step $\tau$ in that zone is selected to be $\tilde{e}_{k}$. 


\section{Relocation strategy "Supply Anticipation"}

Another proactive relocation strategy is the anticipation of the vehicle supply in the network. Such strategies aim at spreading out idle vehicles evenly over the system. This can improve the overall service performance and contributes to service availability in areas with low demand. Supply-anticipatory strategies require fleet regulation, as it hinders the direct competition between the drivers in one fleet. Taxi services with a larger fleet can be regulated according to this strategy by distributing taxis over different taxis stands. For ondemand transport services, this strategy has been simulated mostly on a zonal level (Zhang et al. 2016).

The strategy Supply Anticipation aims at an even dispersal of vehicles across the network based on the amount of available parking spots in the zones. For the time span between the current time step $\tau$ and the horizon time $\tau+\alpha h$, the expected number of parked idle vehicles per zone $K_{z}^{\text {park }}[\tau, \tau+\alpha h]$ is estimated, based on vehicle schedules and the current traffic state. Future scheduling decisions are not considered in this process. From the set of $\zeta$ zones with the least amount of parked vehicles expected at $\tau+\alpha h$, the zone $z$ closest to the current position of the vehicle is chosen, under the condition that there is currently at least one free parking spot available in that zone, as formulated in Algorithm 2. Within that zone, the arc with the largest number of free parking spots at the time step $\tau+\alpha h$ in that zone is selected to be $\tilde{e}_{k}$. Again, a parking spot for the vehicle is immediately reserved on that arc.

\section{Relocation according to "Demand-Supply Deficit Minimization"}

A third proactive strategy combines both perspectives by balancing demand and supply, which aims at balancing the anticipated demand and vehicle supply throughout the service area in order to ensure a high service efficiency. The implementation of this strategy requires fleet regulation in order to guarantee that drivers relocate to locations that are sub-optimal from the driver perspective, but optimal from the system perspective. Various heuristics and optimization approaches aiming at balancing the supply and demand have been simulated, most commonly either for pick-up stations or on a zonal level (Azevedo et al. 2016; Fagnant et al. 2015; Fagnant and Kockelman 2014; Sayarshad and Chow 2017; Zhang and Pavone 2016; Zhang et al. 2015).

The strategy of Demand-Supply Deficit Minimization applied in this study is a combination of the two previous relocation strategies. Idle vehicles are sent towards the zone with the highest supply deficit in relation to anticipated demand. This deficit is defined as the number open requests occurring between the current time step $\tau$ and the horizon time $\tau+\alpha h$ in zone $z$, and the number of idle vehicles at $\tau+\alpha h$ in that zone, similar to the "balance value" applied in (Zhang et al. 2015). The zonal demand-supply deficit is based on the simplifying assumption that open requests located in zone $z$ are dispatched to idle vehicles located within the same zone. This assumption is used in devising the relocation strategy and has no impact on the actual request-vehicle matching by the dispatcher at time step $\tau+\alpha h$.

To determine the deficit value, the number of potentially available vehicles is subtracted from the number of open requests for each zone. Out of the set of $\zeta$ zones with the largest predicted vehicle deficit, thus with the highest deficit value, at time step $\tau+\alpha h$, the one closest to the current vehicle location $e_{k}(\tau)$ is assigned as the destination zone $z^{*}$ for the 
relocating vehicle. In case that no zone is predicted to have a vehicle deficit at $\tau+\alpha h$, the vehicle will relocate within zone $z_{k}$ in which it is currently located. Within the target destination zone for relocation, the arc with the largest number of free parking spots in that zone is selected to be $\tilde{e}_{k}$, as formulated in Algorithm 3. Again, a parking spot for the vehicle is reserved immediately on that arc. In case that none of the arcs in that zone has residual parking capacity, the vehicle is parked in the closest zone with free parking on the arc with the largest number of free parking spots at the current time step $\tau$.

\section{Performance and level-of-service synthesis}

There are several ways of assessing the performance of a relocation strategy, as shown in the literature overview of in Table 1. Which strategy might be chosen depends on the objective that is formulated for services operated by the SAV. Different perspectives may be considered by the various stakeholders, such as the fleet operator, customers, municipalities tendering the on-demand transport services, other road users and residents of the city where such services are operated. The relocation of vehicles impacts the service efficiency, but also service externalities and service equity. Service efficiency can be defined from a user perspective (e.g. in terms of average passenger waiting times per passenger trip) or from a supplier perspective (e.g. in terms of the ratio of vehicle-kilometres-travelled (VKT) without passengers on-board over the total VKT). The externalities of a service operated by SAV are the costs and benefits that affect those not making use of the service, which can be for example its contribution to congestion, undesired environmental effects or the use of public parking facilities. The equity dimension relates to the distribution of benefits and costs of the service over different population groups, notably as varying in their residential location. In terms of benefits, it relates to the variation in service quality as defined for instance in waiting times. In terms of costs, it relates to the distribution of congestion or the spatial pattern of the use of parking facilities. Table 1 provides a brief overview of simulation studies of on-demand transport fleets, the applied relocation strategies in these studies, and the key performance indicators employed in the assessment of the simulation results.

\section{Case study application}

As a simulation environment, the agent-based model MATSim has been used (Horni et al. 2016). The operation of the SAV has been simulated by applying the Dynamic Transport Services module of MATSim (Maciejewski 2016). To analyse the impact of vehicle relocation strategies on the performance of SAV, the operation of SAV is simulated for a case study centred around the city of Amsterdam. The road network has been retrieved from openstreetmap.org (OpenStreetMap Contributors 2018), by superposing a coarser network of arterial roads in the metropolitan area with a more detailed network within the city boundaries (Fig. 3a). For analysing the spatial impacts of the different relocation strategies, the municipal area has been divided into zones based on the 4-digit postal codes, resulting in 82 zones, as shown in Fig. $3 \mathrm{~b}$. The average zone size is $2.64 \mathrm{~km}^{2}$ (range: $0.24-13.88 \mathrm{~km}^{2}$; SD: $2.62 \mathrm{~km}^{2}$ ). The zones used for the relocation strategies are thus not equal in terms of size, population, and parking facilities. However, also the current parking zone in the city of Amsterdam is based on the 4-digit postal code division for residential on-street parking, and on a clustering of 4-digit 


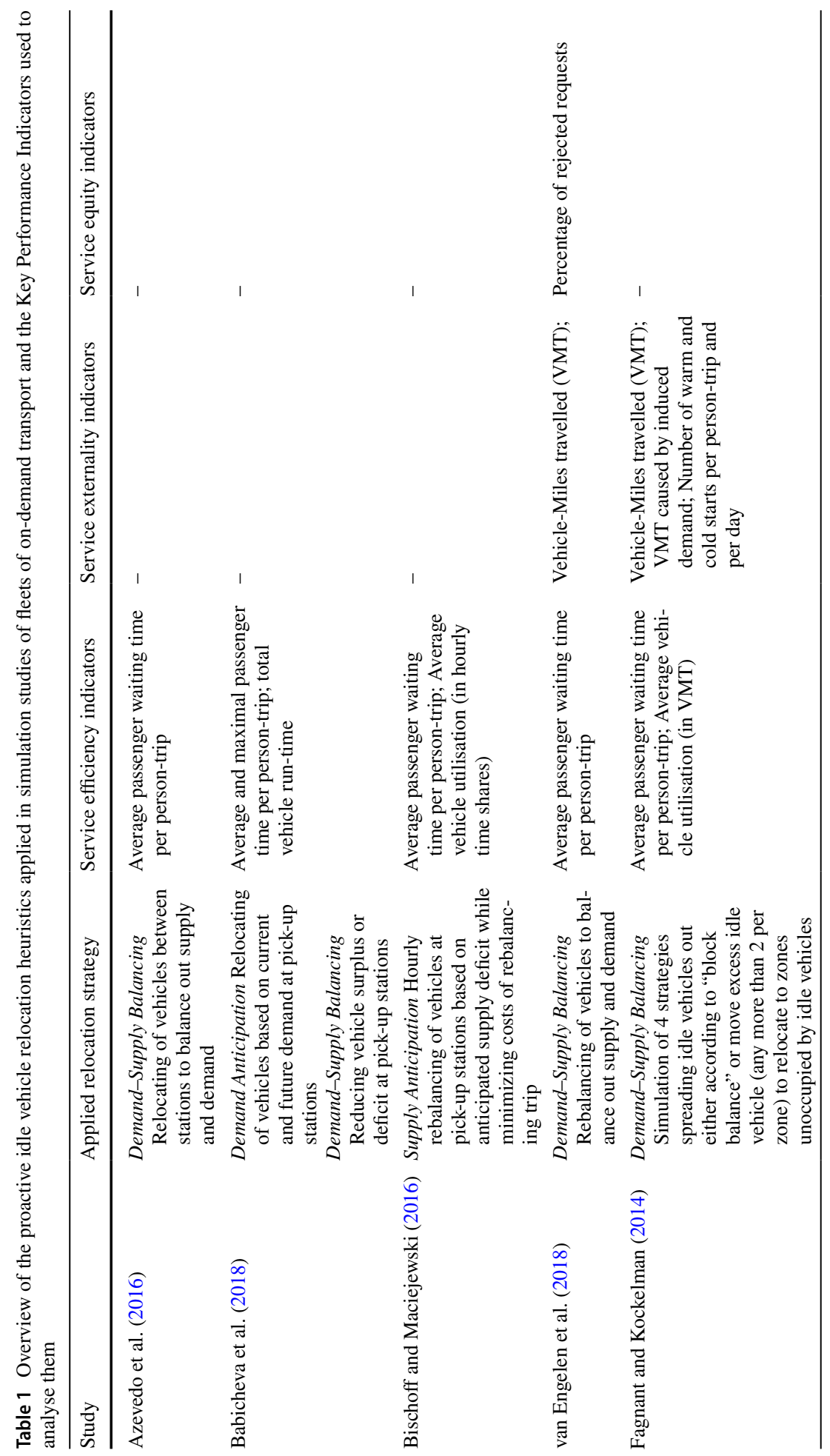




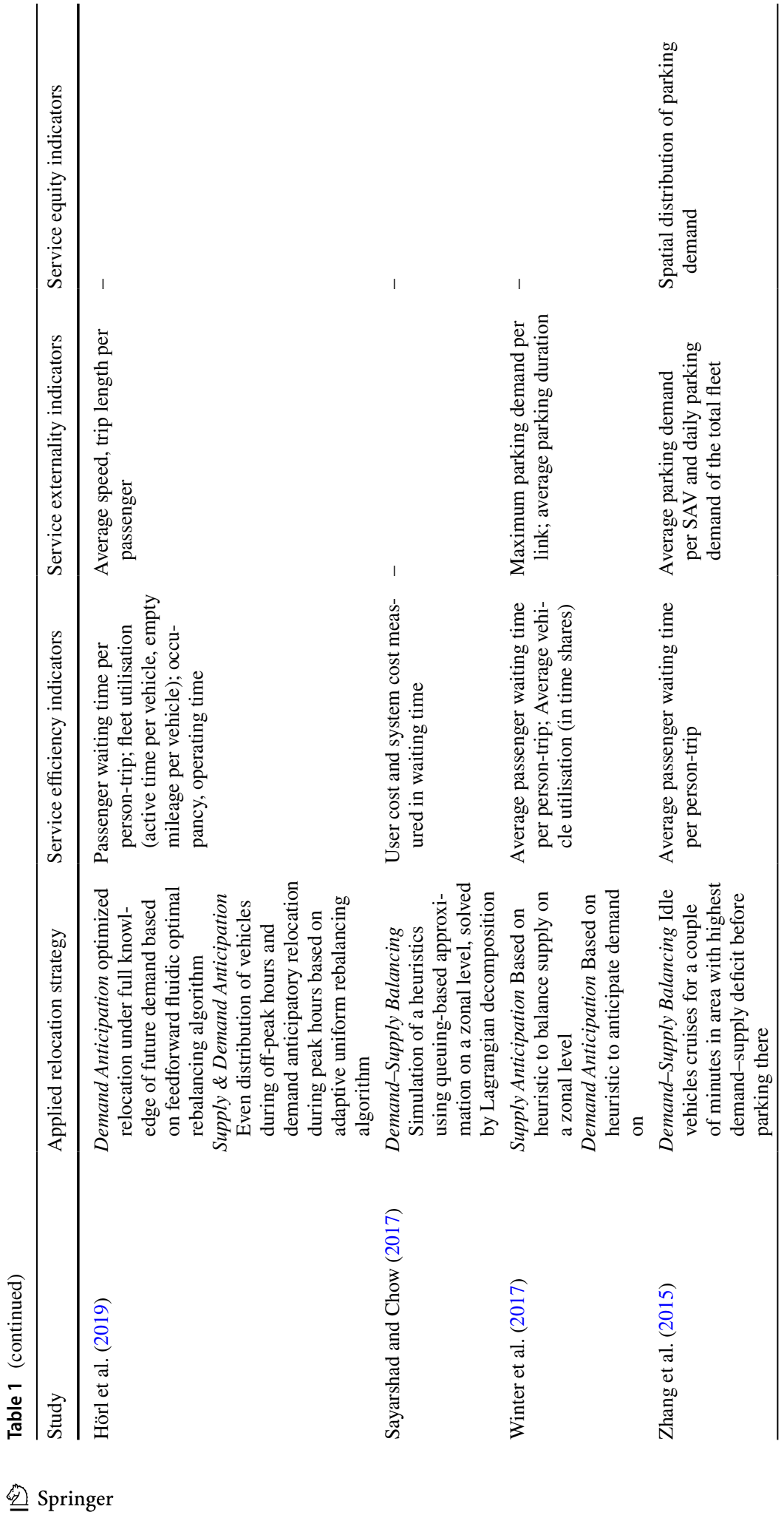




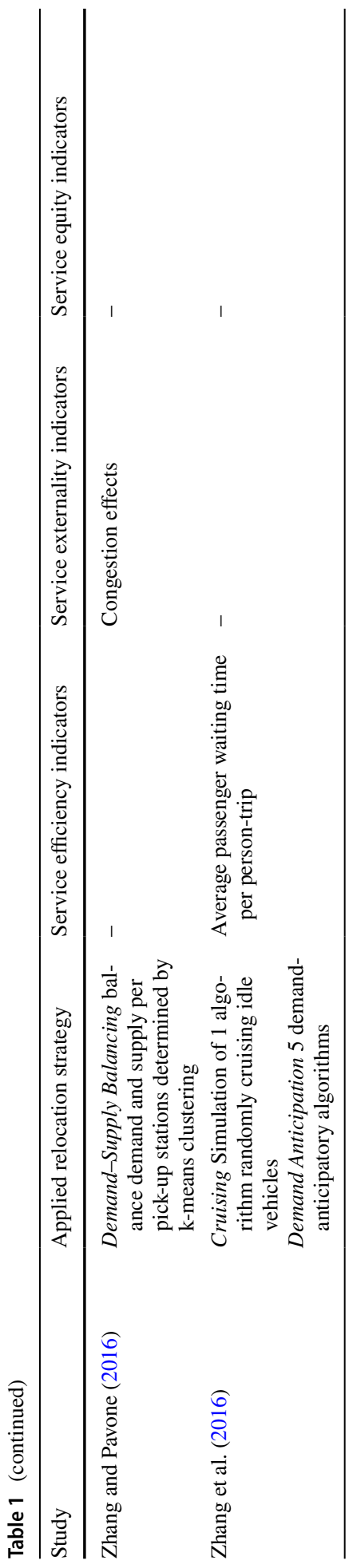




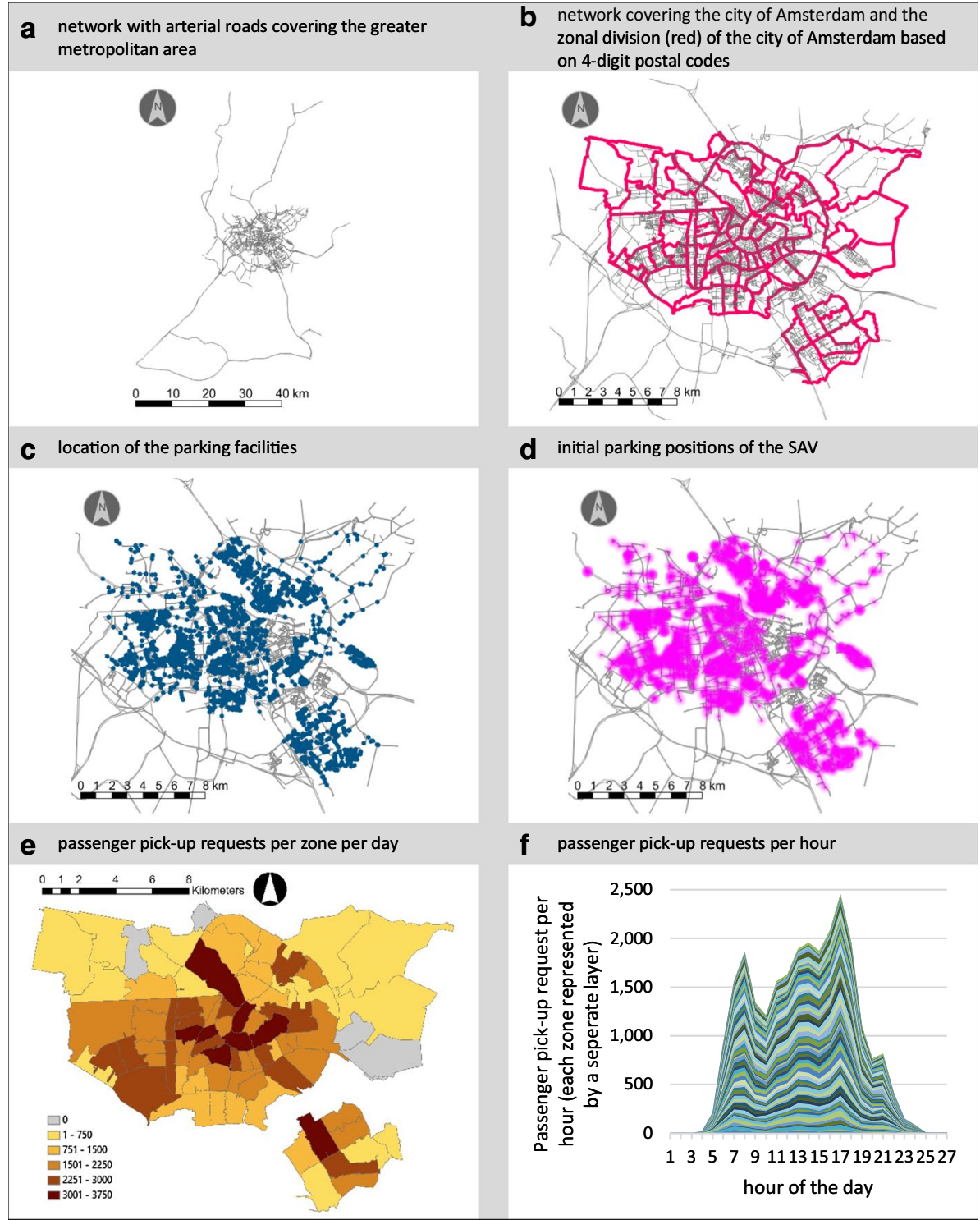

Fig. 3 a Network, b zones, c parking spots $\mathbf{d}$ and initial parking location of the SAV, as well as of the simulated case study of Amsterdam, as well as the e number of passenger pick-up requests of the simulated day per zone and $\mathbf{f}$ passenger pickup requests per hour (each layer represents a zone)

postal codes, grouped to 21 different parking zones, for general on-street parking. For this reason we use the zonal division by 4-digit postal codes as an input parameter to our simulation study as well.

In the following, we describe in more detail the set-up of the simulated scenario, in particular in regard to the simulated agents, their behaviour and their usage of SAV. An overview of all specifications of the Amsterdam scenario is shown in Table 3. 


\section{Travel demand}

The daily activity plans of the agents travelling within the case study network have been specified based on the outcome of the Dutch activity-based model ALBATROSS for the base year 2004 (Arentze and Timmermans 2004). Its outcome is a travel demand model specifying the activities performed by each member of a household, including i.a. the start time and end time of the activity, the 4-digit postal code of the activity and the chosen travel mode to reach the activity location. The Dutch 4-digit postal code areas are quite large (see zonal division shown in Fig. 3). For this reason, we attributed to each activity an actual address within the postal code area at random. The ALBATROSS data set has been reduced to households in which at least one household member performs at least one activity within the municipality of Amsterdam. For computational reasons, we simulate only $20 \%$ of the population, therefore each agent is weighted by a factor of five in the simulation. In doing so, we follow common practice (see also Bischoff and Maciejewski 2016b). This results in a total of 767,495 agents (represented by 153,499) who perform a total of $3,776,805$ activities on a single day and move either by car, public transport or active mode (walking and cycling combined). The majority of the agents are based in Amsterdam, but a substantial share arrives from surrounding suburbs and from nearby towns situated in the greater metropolitan area. The altered data set used in this study and a detailed description of how it has been derived is publically available (Winter and Narayan 2019).

\section{Specification of SAV and their infrastructure needs}

Since automated on-demand transport services are not operational as of now, assumptions concerning their operational and technical specifications, as well as the assumptions on the passengers' reception of such services remain speculative for the moment. For this reason, a simple scenario has been drawn regarding the technical and operational specification of SAV and their according infrastructural needs. The assumptions made on AV technology and infrastructure needs are reduced in complexity so that the simulation results remain traceable and the analytical focus can be put on the relocation strategies.

In this study, SAV are offered as an additional mode alternative to private car, public transport and active modes. In terms of vehicle technology, SAV are regarded to be similar in their performance to private cars, they achieve thus the same driving speeds and have the same physical dimensions. In this study, SAV and private cars share the same road infrastructure, it is such a simulation of mixed traffic. In operational terms, the SAV are assumed to be operated as a centrally dispatched fleet which allows for sequential vehicle sharing. Car-pooling, i.e. simultaneous vehicle sharing, is thus not considered in this study. Vehicles are assumed to fully comply to the dispatcher and operate in a collaborative scheme. In regard to their infrastructural needs, it is assumed that they share the road infrastructure built for private cars and can drive on all links of the road network.

To test the impact of the different relocation strategies, the fleet size has been set to 12,500 vehicles, which leads to an average passenger-waiting time of 4 min-a value which we selected to represent an acceptable level of service. This fleet size for SAV is approximately $2 \%$ of the simulated fleet size of private vehicles. For these vehicles, 15,000 curbside parking spots are reserved throughout the network within the limits of the city area, their location is shown in Fig. 3c. We generated these parking spots per link-arc based on the link length, which therefore determines the storage capacities for idle vehicles per 
link. These generated parking spots are located in the middle of arcs representing residential streets situated within the city boundaries on streets with a maximum allowed speed of $50 \mathrm{~km} / \mathrm{h}$. At the beginning of the simulated day, the SAV are parked randomly on the dedicated parking facilities, as shown in Fig. 3d. The amount of dedicated parking spots has been selected so that sufficient parking space is provided to the SAV at all times throughout the simulation, and so that in addition some extra space is available to efficiently park the vehicles according to the relocation strategies. In this scenario, parking spots can be allocated and reserved by the same central dispatcher, who also performs the request dispatching. We capped the size of the set of candidate zones considered for relocation to three $(\zeta=3)$.

\section{Behavioural model and model specifications}

The modal split present in the reduced ALBATROSS data set is not aligned with the modal split observed for the city of Amsterdam based on all trips taken within the city as well as trips with either their origin or destination within the city boundaries (Gemeente Amsterdam 2016). To overcome this, the plans of the agents have been calibrated by simulation based on the co-evolutionary learning process implemented in MATSim until a modal split similar to the one observed for the city of Amsterdam (including walking and cycling, which account for large shares of trips performed) has been achieved. The calibration has been performed under the conditions that the daily travel pattern remains showing two demand peaks due to commuting and that all agents reach their final destination within the simulated period. While the Amsterdam scenario has been carefully calibrated to reproduce the actual local overall modal shares, the simulated scenario has not been calibrated for more detailed traffic and travel data.

Over time, MATSim agents can alter their daily plans following a set of day-to-day learning rules. The MATSim-specific settings in regard to this simulated learning-behaviour are presented in Table 3. After a completed simulation run of one day, agents either select their next plan from a set of plans they have memorized from previous simulation runs based on the plans' scores, or alter parts of their plan according to pre-defined planinnovation rules (see Table 3). The learning behaviour simulated in MATSim is based on the concept of utility. The utility of performing an activity is described by the activity duration, the waiting time in case of arriving too early, a potential delay, a potential early departure and the potential reduction of the desired time spend on the activity (Nagel et al. 2016). The coefficient for the utility of performing an activity, $\beta_{\text {duration }}$ is based on the value of the average hourly wage in Amsterdam in the year 2017, which is 16.25 Euro per hour (Gemeente Amsterdam 2018). The coefficient for arriving late is weighed three times as much as $\beta_{\text {duration }}$, following the standard MATSim settings and the findings in Börjesson et al. (2012). The disutility of travelling depends on travel time and travel costs. The coefficient for travel time $\beta_{\text {travel_time, } m}$ is mode specific, while the one for travel cost $\beta_{\text {travel_cost }}$ is generic, based on the assumption that costs are perceived in a rational manner. Additional mode-specific preferences are represented by the Alternative Specific Constants $\left(A S C_{m}\right)$. The cost parameters and the mode-specific constants for travel time for the modes car, public transport, cycling and walking as well as the cost parameters are, where possible, based on values reported in literature (van Ommeren et al. 2012) and are presented Table 3.

The values for $\operatorname{cost}_{S A V}$ are based on values reported for the simulation of comparable services, which range between $14 €$-cent $/ \mathrm{km}$ and $91 €$-cent $/ \mathrm{km}$, with most studies settling at price similar to the one used in our study (see Bösch et al. 2018; Gurumurthy 
et al. 2019; Simoni et al. 2019). The values for the perceived utility of SAV have been set to be the same as the ones for private car, since the way we envision this transport service is most similar to the one of the mode "car" in this model: passengers are moved inside a motorized vehicle, which is not shared with strangers, and provides an on-demand door-to-door transport service. Currently, the state-of-the-art discrete choice models comprising the choice between SAV and the other modes included in this model are not unequivocally enough to make an assertive statement about the relative difference in the perceived utility of SAV and car (Ashkrof et al. 2019; de Looff et al. 2018; Simoni et al. 2019). However, the specified behavioural model serves the purpose of creating a test-bed in which strategies for idle vehicle relocation for SAV can be analysed in regard to their service efficiency, externalities and equity. This can be achieved with the simplified model described in Tables 2 and 3. Nevertheless, given the many uncertainties linked to the user preferences towards vehicle automation in general, and shared automated vehicles in particular, we refrain from analysing the impact of the relocation strategies on mode shifts and mode shares. The latter should be included in such an analysis once more reliable mode-choice models for SAV are available. We should point out that in our case study the applied behavioural model is set in the (Dutch) context of the case study and hence the SAV is primarily in competition with the longer-distance modes 'private car' and 'public transport'. As a result, the average distance for trips taken in SAV is around 12 kilometres, which lies well above the 7.5 kilometres which mark the threshold distance above which the Dutch population tends to switch from active modes (walking and cycling) to car (Ministry of Transport Public Works and Water Management 2009).

For this reason, we suppressed mode changes in the final model used to test the relocation strategies. To able to do so, we split the simulation process into three parts: (1) In the first calibration phase, we let agents incorporate SAV into their daily plans in the course of 76 repeated simulations of one day. During the cause of these simulated days, agent mode choice behaviour stabilized, leading to a modal share of $4 \%$ for SAV, which equals approximately 130,000 trips performed using SAV per day (Table 3). (2) The resulting plans of the final simulated day have been used as an input in the second simulation phase, in which the same day has been simulated in 16 runs. (3) The output of this second round has been used as an input for the final simulation, in which the same day is simulated only twice, while suppressing any mode choice innovations. The second simulation phase has proven to be necessary due to a particular feature inherent to MATSim's Dynamic Transport Services module, which uses the exponential moving averages of link travel times over all iterations of a simulation for its dispatching algorithm. Without the intermediate step, the output of the last day of the first calibration phase would not lead to the same results of the first day of the final simulation. The applied solution to this problem has also been suggested in (Maciejewski and Bischoff

Table 2 Constants and coefficients specified for the utility function formulating the mode choice behaviour of the agents

\begin{tabular}{lccll}
\hline Mode & $A S C_{m(q)}$ & $\beta_{\text {travel_time,m(q) }}$ & $\beta_{\text {travel_cost }}$ & cost $_{q}$ \\
\hline Car & 0.0 & -10.7 & 1 & $30 €$-cent $/ \mathrm{km}$ \\
Public transport & -8.3 & -6.65 & 1 & $25 €$-cent $/ \mathrm{km}$ \\
Cycling & -1.0 & -10.7 & 1 & $0 €$-cent $/ \mathrm{km}$ \\
Walking & 0.3 & -6.65 & 1 & $0 €$-cent $/ \mathrm{km}$ \\
SAV & 0.0 & -10.7 & 1 & $30 €$-cent $/ \mathrm{km}$ \\
\hline
\end{tabular}



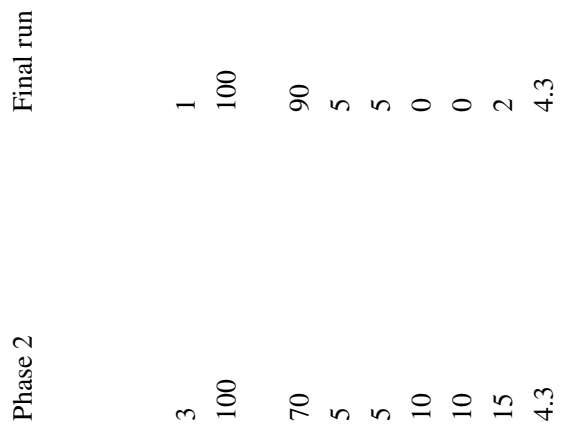

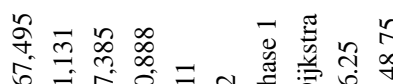

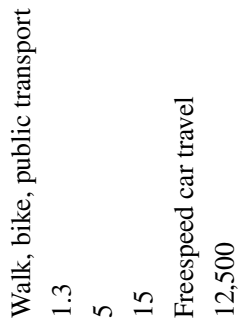

ஸे

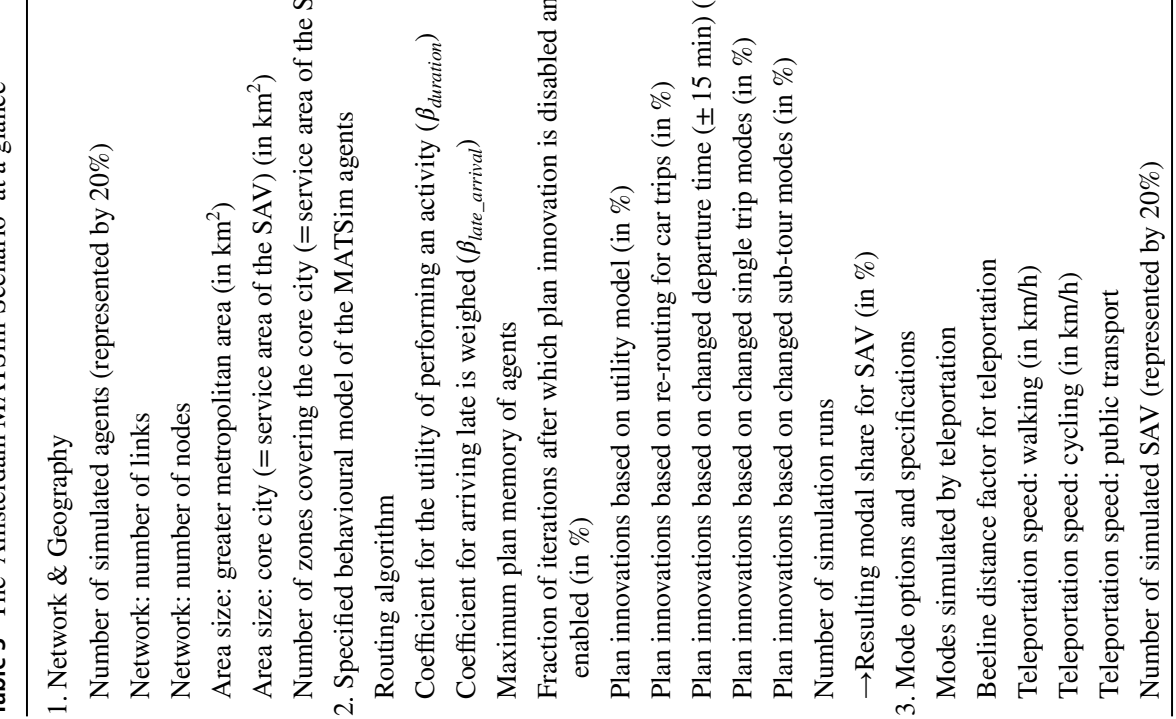




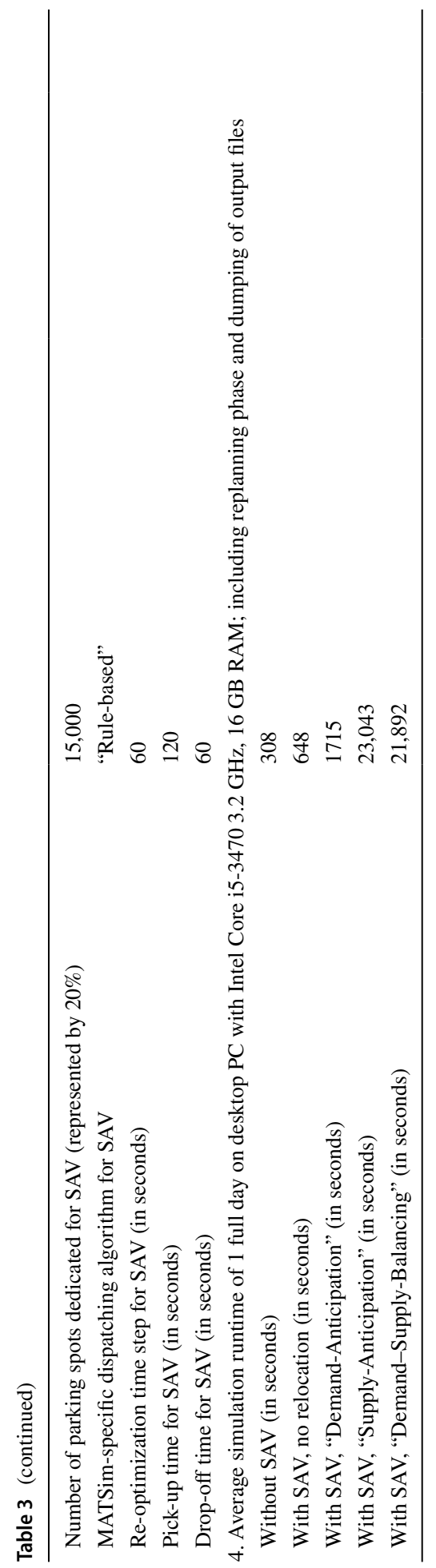


2018). The demand for SAV is shown on a zonal level in Fig. 3e, the temporal distribution of the requests for SAV are shown in Fig. 3f, in which the requests per hour per zone are stacked up on top of each other in layers.

During the learning phase of the agents, the relocation strategy of "demand-anticipation" has been applied to capture the appropriate agent learning behaviour as a response to SAV service that is subject to parking restrictions. We opted for this strategy for computational reasons, as this strategy shows the shortest computational times (see Table 3). The resulting plans of this simulation are used as an input to all following simulations testing the different relocation strategies. The input to the scenarios simulating the different relocation studies is thus the output of the simulations performed in the initialisation phases. It contains a set of agents and their activity schedules for the simulated day, including their travel behaviour. For the simulation of the relocation strategies for SAV, these plans are not altered any further, the demand for SAV is thus kept inelastic.

\section{Results}

In the following, the results for the three relocation strategies of Demand Anticipation, Supply Anticipation and Demand-Supply Balancing are assessed for three categories of key-performance indicators (KPI): (1) service efficiency, (2) service externalities and (3) the service provision equity. We also include into this discussion the results for the scenario Remain, for which vehicles simply wait at their latest drop-off location, irrespective of the availability of parking facilities. The Remain scenario is thus not a valid representation of the real-life constraints caused by the scarcity of road-space and parking-space. For this reason, we focus on the comparison between the three scenarios in which idle vehicles have to relocate according to one of the relocation heuristics (Demand Anticipation, Supply Anticipation and Demand-Supply Balancing), and not on the comparison between a situation with and without the relocation of idle vehicles. However, we provide results for the scenario Remain in order to allow a comparison also to other simulation studies of SAV with this feature. All discussed KPIs are based on the average results of 4 runs. The number of necessary runs per relocation strategy has been determined by a two-sided $t$ test between means with a $95 \%$ confidence interval.

\section{Service efficiency}

The service efficiency is measured in KPI describing the quality of service from a passenger's perspective, as well as KPI showing how efficiently the transport service can be operated. For an overview of these KPI, see Table 4, as well as Figs. 4 and 5.

\section{Waiting times}

The average passenger waiting time $t_{S A V_{-} \text {wait }}$ is with $3.5 \mathrm{~min}$ the lowest for the strategy Demand-Supply Balancing. The highest average waiting time with 4.6 min occurs for the strategy Demand Anticipation. The average waiting time for the Supply Anticipation strategy lies with 3.6 min close to the one of the Demand-Supply Balancing strategy. All three relocation strategies increase the passenger waiting times in comparison to the Remain scenario, for which the average passenger waiting time is $2.2 \mathrm{~min}$. In regard to $95 \%$ percentile of the passenger waiting times $t_{S A V_{-} \text {wait_ } 95 \%}$, the same trend than for the average passenger 


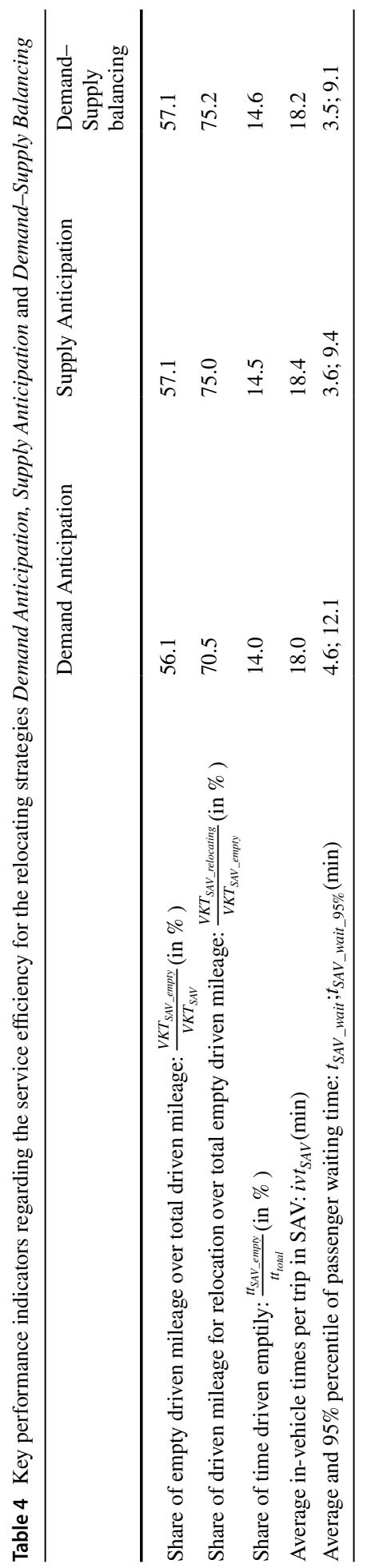




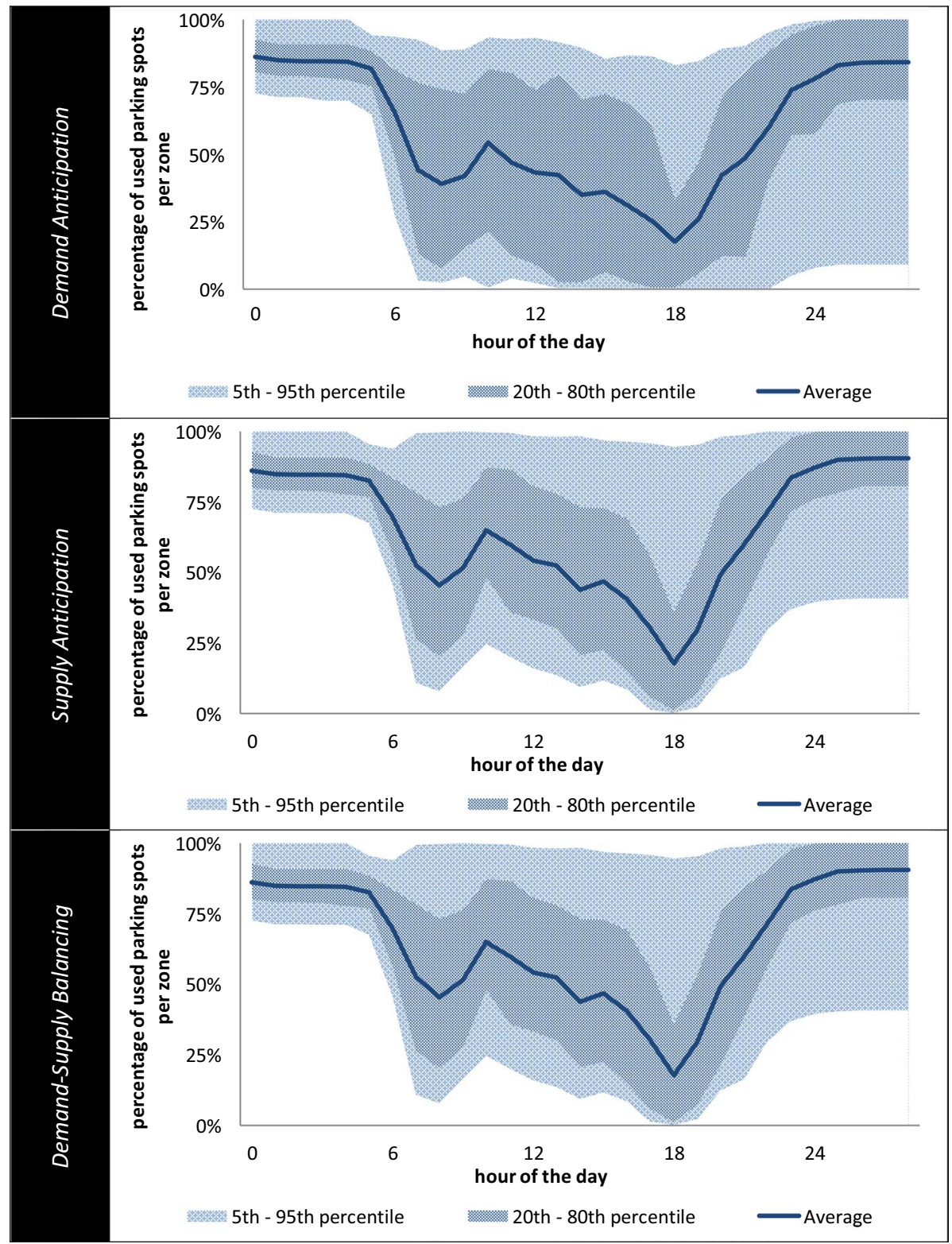

Fig. 4 Average zonal parking usage (solid line) over the course of a simulated day. The 5th-95th percentile and 20th-80th percentile is shown by the shaded areas

waiting time can be observed: with 12.1 min the highest value is reached for the strategy Demand Anticipation, with 9.1 min the lowest value for the strategy Demand-Supply Balancing, closely followed by the one for Supply Anticipation.

We did not set a cap on the maximum waiting times, therefore no requests have been declined or cancelled by passengers. This leads to a maximum passenger waiting time of 


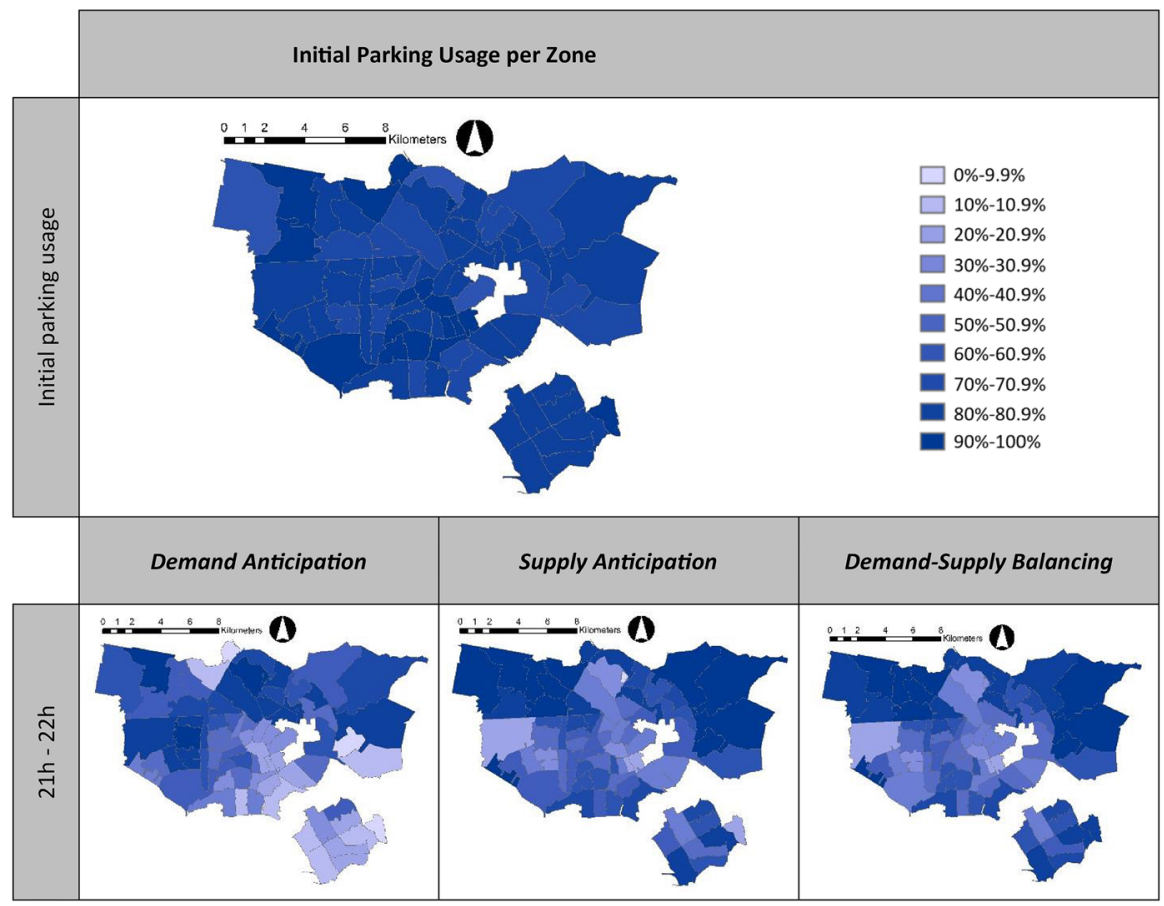

Fig. 5 Zonal parking space utilisation rate for the three relocating strategies Demand Anticipation, Supply Anticipation and Demand-Supply Balancing after the evening peak hour (21-22 h). The initial parking usage per zone is shown at the top

189 min in the scenario Remain. For the scenarios with idle vehicle relocation, the longest maximum passenger waiting time is reached for the strategy Supply Anticipation (278 min), followed by the strategy Demand Anticipation (271 min) and Demand-Supply Balancing (252 min). These very long waiting times cannot be interpreted as the expected maximum waiting times for an on-demand service operated by SAV, as it is not realistic that passengers would wait several hours for their ride to arrive. But these values show the extent to which the different strategy disadvantage passengers in different areas, which is discussed in more detail in "Service provision equity" section.

\section{Empty driven mileage}

The total driven mileage for a fleet of SAV is composed of the VKT with passengers onboard as well as VKT driven emptily. The ratio between the VKT with and without passengers on-board is an important KPI for the efficiency of the service. In our scenario, the average trip length for trips taken in SAV is approximately 12 kilometres, but the total VKT travelled per trip is a threefold of this once idle vehicle relocation is introduced. In the course of a day, SAV are driving emptily ( $\left.V K T_{S A V \_e m p t y}\right)$ for two different purposes: (a) moving from the latest drop-off location to the assigned parking spot leads to empty VKT due to relocation $\left(V K T_{S A V_{-} \text {relocating }}\right)$ and (b) moving from the parking spot to the next pickup location. A leading KPI to measure the efficiency of the operation of an SAV service is 
the share is of the empty driven mileage $V K T_{S A V \_ \text {empty }}$ of the total driven mileage $V K T_{S A V}$, which varies between 56.1 and $57.1 \%$ for all three relocation strategies. If vehicles are not relocated, as simulated in the scenario Remain, this ratio drops to $10.2 \%$.

When looking in more detail at what causes the empty VKT, it can be observed that the relocation strategy Demand Anticipation has with $70.5 \%$ the lowest share of empty

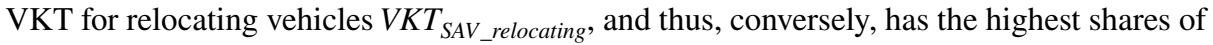
empty VKT for approaching passengers at their respective pick-up locations. The strategies Supply Anticipation and Demand-Supply Balancing have with $75.0 \%$ and $75 \%$, respectively, a higher share of empty VKT caused by relocation, and conversely fewer empty VKT caused by moving from their parking location to the pick-up locations of their next customer.

\section{Trip times}

The time for a trip in an SAV experienced by a passenger is a combination of waiting time, the time it takes to enter the vehicle (set to $120 \mathrm{~s}$ in the simulation), the in-vehicle time and the time it takes to exit the vehicle (set to $60 \mathrm{~s}$ in the simulation). For the scenario Remain, the average in-vehicle time per trip $\left(i v t_{S A V}\right)$ is $15.4 \mathrm{~min}$, and the average trip time is $20.6 \mathrm{~min}$. When introducing idle vehicle relocation, the average trip times increase by factor 1.2, with Demand-Supply Balancing leading to an average trip time of $24.8 \mathrm{~min}$, followed by Supply Anticipation (25.0 min) and Demand Anticipation (25.3 min). The difference in trip time between the strategy Demand Anticipation and Demand-Supply Balancing is $57 \mathrm{~s}$, which translates in our scenario to a difference of 2042 passenger-hours saved for users of the SAV in case of the strategy Demand-Supply Balancing. The differences in in-vehicle time originate solely from different levels of congestion, which is discussed in more detail in the "Congestion" section, since the demand is kept inelastic for the analysed case study.

\section{Service efficiency: summary}

Concerning service efficiency, it can be concluded that the strategy Demand-Supply Balancing leads to the shortest average passenger waiting times, which also leads to the shortest total trip times for the simulated case study. This comes however at the cost of longer in-vehicle travel times, which are a result of congestion effects caused by relocating vehicles to areas with high demand, as well as congestion elsewhere in the network due to vehicles spreading out in zones with an undersupply of vehicles. These local congestion effects due to vehicle bunching are the strongest for the strategy Demand Anticipation, which is also the strategy with the longest average passenger waiting time. However, when it comes to the shares of empty driven mileage or the time spend on relocating, is the strategy Demand Anticipation the most efficient for the simulated case study, and the strategy Demand-Supply Anticipation the least efficient one.

\section{Service externalities}

The externalities of SAV relocation strategies are analysed for three aspects: (1) the average driving speed as a proxy for congestion, (2) the total driven mileage as a proxy for 
Table 5 Key Performance Indicators regarding the service externalities for the relocating strategies Demand Anticipation, Supply Anticipation and Demand-Supply Balancing

\begin{tabular}{lll} 
Demand Anticipation & Supply Anticipation & $\begin{array}{l}\text { Demand- } \\
\text { Supply } \\
\text { balancing }\end{array}$ \\
\hline 39.2 & 39.2 & 39.1 \\
$38.9 ; 39.6$ & $39.3 ; 39.1$ & $39.0 ; 39.2$ \\
3519 & 3610 & 3608 \\
\hline
\end{tabular}

energy consumption and potential emissions and (3) the spatial consumption of curbside parking space by SAV. For an overview of these KPI, see Table 5.

\section{Congestion}

As already pointed out in the previous section, the different relocation strategies lead to different levels of congestion. In this simulation, potential congestion effects caused by pick-up and drop-off situation or potential lower flow density caused by mixed traffic with automated and non-automated vehicles are not included, and the presented values might be therefore an underestimation of congestion SAV might cause.

The average driving speed of the SAV $\left(v_{S A V}\right)$ in the scenario Remain is $46.4 \mathrm{~km} / \mathrm{h}$. When forcing vehicles to relocate when idle, the $v_{S A V}$ goes down by about $17 \%$ (see Table 4 ). Using the average speed as a proxy for congestion, it can be concluded that vehicle relocation causes undesired externalities in the form of additional disturbances in the network flows. To understand better how and where the different relocation strategies can cause congestion, we analyse the driving speeds of SAV with and without passengers on-board separately. The average driving speed for SAV for the scenario Remain is $46.0 \mathrm{~km} / \mathrm{h}$ with passengers on board $\left(v_{S A V_{-} I V T}\right)$ and $50.0 \mathrm{~km} / \mathrm{h}$ without passengers on board $\left(v_{S A V_{-} e m p t y}\right)$. When introducing idle vehicle relocation, in particular $v_{S A V_{-} e m p t y}$ goes down (roughly by $25 \%$ for all relocation strategies compared to the scenario Remain), indicating that vehicles driving empty to and from their parking locations experience (and create) more congestion than those serving passenger request. The impact on $v_{S A V_{-} I V T}$ on the other hand is less strong, with the percentage difference being approximately $15 \%$ for all strategies compared to the scenario Remain. Looking in more detail at the differences between the relocation strategies, it can be observed that for the strategies that relocate idle vehicles closer to anticipated future demand (Demand Anticipation and Demand-Supply Balancing), $v_{S A V_{-} \text {empty }}$ is faster than $v_{S A V_{-} I V T}$, and that the differences between these two speeds are more pronounced than for the strategy Supply Anticipation. This is a direct result of the boundaries set by the relocation algorithms, which leads in the case of anticipated demand to vehicle accumulation in the areas with the highest demand levels. As a consequence, idle vehicles are blocking each other when departing from the zones with high demand. The strategy Supply Anticipation, on the other hand, creates less of locally concentrated congestion, but slows down traffic flows more evenly in the network. The strategy Demand-Supply Balancing combines, in regard to congestion effects, the worst of both strategies and leads consequently to the lowest average driving speed. 


\section{Driven mileage}

In this study, the discussion of the effects of vehicle relocation of SAV on energy consumption and emissions is deliberately kept on an abstract level. No assumptions on the source of vehicle propulsion for the SAV is made, thus also no assumption on the magnitude of energy consumption, fine dust matter in the form tyre debris (see Kole et al. 2017), noise pollution (see Campello-Vicente et al. 2017) or other emissions can be made. The impact of the relocation strategies is instead based on the total driven mileage as a proxy, which can be used as input to any traffic emission estimation model. The total mileage driven by SAV is presented in Table 5 .

For the scenario Remain, the total $V K T_{S A V}$ for the entire fleet is $1,707,415 \mathrm{~km}$, which corresponds to an average of $13.2 \mathrm{~km}$ driven per trip served by SAV. When introducing idle vehicle relocation, the total VKT increase with more than factor 2 . When applying the strategy Demand Anticipation, the total $V K T_{S A V}$ is $3,518,975 \mathrm{~km}$, translating to $27.2 \mathrm{~km}$ driven per served passenger trip. The strategies aiming at spreading out idle vehicles more increase the total driven mileage even further, with $V K T_{S A V}=3,609,493 \mathrm{~km}$ for the strategy Supply Anticipation and $V K T_{S A V}=3,607,769 \mathrm{~km}$ for the strategy Demand-Supply Balancing, translating to approximately $27.9 \mathrm{~km}$ driven per served passenger trip. The results of these additionally VKT are overall lower driving speeds, as discussed in the previous section.

\section{Parking space consumption}

As a third aspect of service externalities, the curbside parking consumption is analysed. This analysis is conducted at a zonal level. For each zone, the parking space utilisation rate has been determined on a minute basis and is averaged per hour, allowing to trace the parking use over time.

In Fig. 4, the course of the hourly parking usage averaged over all zones is shown. The average parking usage follows a similar pattern over the course of the day for the three relocation strategies, and averages to about $65 \%$ for all strategies for the whole day. However, the distribution of the parking usages for the strategy Demand Anticipation differs strongly to the one for the strategies Supply Anticipation and Demand-Supply Anticipation, which can be clearly seen when comparing the range of the 5th-95th percentile and the 20th-80th percentile. When applying Demand Anticipation, the spatial distribution of idle vehicles follows the demand patterns simulated in the case study, which is not evenly distributed, as shown in Fig. 3e, f. This leads to parking facilities in zones with high demand getting fully used, while parking spots in zones with lower demand remain unused. This effect is particularly strong during the off-peak hours, thus the periods in which most vehicle relocations happen, which increases the variance in spatial distribution further.

The parking usage depends on the shape, size and parking capacity of the zones, and is thus case-specific. For this reason, it is important to not just look at the average usage over zones and its respective variance, but also look at the distribution of zonal waiting times in the heat maps shown in Fig. 5. These show that the strategy Demand Anticipation leads to a more unbalanced distribution of parked vehicles throughout the city than the other two strategies by concentrating idle SAV in high-demand areas in the North of the city, following the demand pattern shown in Fig. 3e.

In Fig. 5, the hourly average of parking usage is shown for two moments in time: the initial parking usage in the first hour of the simulated day and the parking usage after the 
evening peak in the 21 st hour of the simulated day. We present this hour of the day in more detail, as it is the first off-peak hour following the evening peak and thus shows most relocation actions. This leads also to the most unequal distribution of idle vehicles in the evening hours (see Fig. 4), meaning that during this hour the differences for this KPI between the different relocation strategies are the most striking. The strategy Demand Anticipation leads to more parked vehicles in the centre and the West of the city, while Supply Anticipation and Demand-Supply Balancing lead to more parked vehicles in the South and East of the city, and overall to a more even distribution of idle vehicles in the city area.

\section{Service externalities: summary}

In regard to undesired externalities, it can be concluded that the strategy Demand Anticipation leads to idle vehicle congregating in high demand areas and thus causes local congestion and an uneven usage of the parking facilities, but also creates less congestion in the network overall and contributes the least to energy consumption and emissions. The strategy Demand-Supply Balancing, on the other hand, is less favourable for reducing undesired emissions and contributes more to congestion in the network than Demand Anticipation. The strategy Supply Anticipation causes the highest number of VKT, but outperforms the strategy Demand-Supply Balancing in regard to congestion.

\section{Service provision equity}

In this study, the service provision equity of the on-demand transport service operated by SAV is analysed in regard to the distribution of waiting times. As a KPI, the Gini-index for all passenger waiting times $G_{\text {wait }}$ and for the zonal average passenger waiting times $G_{\text {wait }, z}$ is used, which are shown in Table 6. The Gini-index (Gini 1912) is a well-known measure of inequality, often used in an economic context. A concise description of the Gini-coefficient and its applications in the field of transport as a means to measure inequality of accessibility can be found in (Ben-Elia and Benenson 2019). In short, the Gini-index is a measure of distribution, ranging from 0 to 1 , with 0 indicating perfect equality and 1 perfect inequality. The closer the Gini-coefficient is to 0 , the more passengers experience similar waiting times. A high Gini-coefficient, in turn, represents a situation where people differ substantially in their experienced waiting times. In this study, we use the Gini-coefficient only to describe the distribution of waiting times over the whole population, without taking into account the differences between people, such as income or mobility needs.

\section{Waiting time distribution}

The Gini-index for the passenger waiting times $G_{\text {wait }}$ for the scenario Remain is 0.453 , and for the average zonal passenger waiting times $G_{\text {wait }, z}$, the Gini-index is 0.215 . For the scenarios with idle vehicle relocation, the highest Gini-index, and thus the most unequal distribution for passenger waiting times occurs when applying the strategy Demand Anticipation $\left(G_{\text {wait }}=0.554\right)$. This is also the strategy that leads to the longest average waiting times (Table 4), which shows that the gains in overall reduced waiting times by placing vehicles strategically in anticipation of future demand not only comes at the cost of overall longer waiting times for passengers, but that these are also particularly unequally distributed by systematically disadvantaging passengers in zones with lower demand. This can be also be seen in the spatial representation of averaged waiting times, where it obvious 


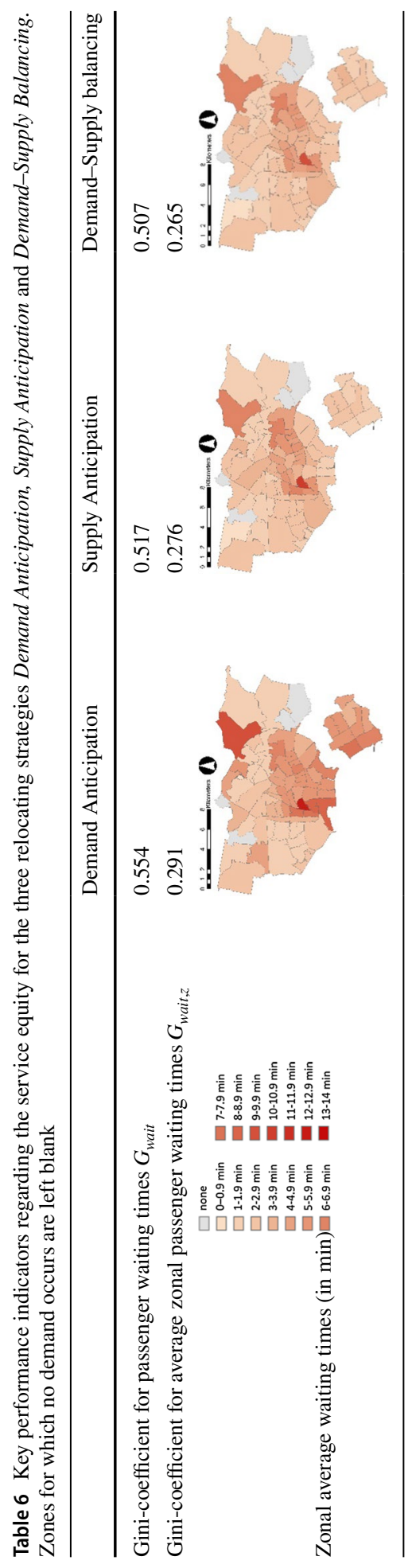


that passengers in the West of the city benefit from shorter average zonal waiting times compared with those in the North-East and South. The lowest Gini-coefficient for the total waiting times is achieved with the strategy Demand-Supply Anticipation, which is also the strategy leading to the lowest average waiting times (Table 4). In the simulated case study, a more equal distribution of all waiting times leads thus to higher efficiency in the service operation in regard to average waiting times.

\section{Service provision equity: summary}

Regarding the service provision equity, it can be concluded that the strategy Demand-Supply Balancing not only leads to the shortest waiting times on average, but it also leads to the highest service provision equity. When positioning idle vehicles close to future requests, as done for the strategy Demand Anticipation, the short approach times which can be achieved for customers in high-demand zones come at the cost a those living in low demand zones and who consequentially experience very long waiting times. This reduces not only the service provision equity, but also increases the average passenger waiting time.

\section{Discussion and conclusion}

\section{Impact of idle vehicle relocation}

With shared mobility and on-demand transport services gaining steadily more ground, and the automation of vehicles pushing into the field of transport as the next 'disruptive' technology, the need for reliable simulation studies for testing operational strategies for AV and SAV is increasing. This study has shown that an important component of operating such vehicles in a large fleet is the relocation of idle vehicles during off-peak hours. In the urban context, space for idle vehicles is scarce and parking is often constrained. The relocation of idle vehicles is thus a necessary consequence of real-world parking constraints. For this reason, it is important to test for the impact of different relocation strategies when introducing SAV to a city, and specify them for example in a tendering process or when tailoring curbside management strategies. Including vehicle relocation under parking constraints to the simulation of the operation of SAV is thus an important step to increase the realism of the simulation and consequently improve the analysis of such transport services. For this reason, it is not the comparison between the Remain strategy and the simulated relocation per-se the subject of analysis, but rather primarily the comparison between the different relocation strategies, which take the real-life constraints caused by the scarcity of roadspace and parking-space into account. However, there are two main insights gained from referencing the scenarios with pro-active relocation strategies against a situation where parking constraints are not accounted for (i.e. Remain): (1) The relocation of idle vehicles does not necessarily lead to performance gains for a fleet vehicles providing on-demand transport services. As a consequence, there is a risk of overestimating the performance of such fleets in simulations in case the relocation of empty vehicles is not accounted for. (2) Relocating idle vehicles in a pro-active manner might be outperformed by reactive relocation strategies in regard to the service efficiency and the total driven mileage. Since this finding is case-specific and depends on the spatial and temporal distribution of the demand, more research is required in order to determine the conditions under which it is favourable to apply reactive or proactive relocation strategies. A remain strategy can be feasible in 
certain areas where ample curbside parking facilities are available, especially if those are to be prioritized for SAV. Such areas are typically located in the outskirts of cities, which means that pro-active relocation strategies might be particularly suitable for city centres or other areas with little space and high demand. In this study, three pro-active relocation heuristics based on zonal parking availability are compared to each other, in terms of service efficiency, service externalities and service provision equity, for a case study based on the city of Amsterdam, the Netherlands. Performance differences could be detected regarding the quality of the offered service (average passenger waiting times, average trip times), the impact on traffic (local congestion, total driven mileage), the parking space usage and the spatial service provision equity (distribution of passenger waiting times).

The strategy Demand Anticipation, which relocates vehicles to zones with the highest number of upcoming requests, leads to short average passenger waiting times in these zones. This, however, comes at the cost of passengers experiencing longer waiting times in zones with less demand. This relocation strategy leads, overall, to the longest average passenger waiting times, and to the least equal service provision. This strategy also causes bunching of vehicles in areas close to demand hotspots, which causes local congestion and high usage of parking facilities in those areas. As a consequence for this particular case study, vehicles drive shorter distances when empty, leading to more efficient usage of the rolling stock, and cause less overall congestion in the network compared to the other two relocation strategies.

The strategy Supply Anticipation aims at distributing idle vehicles evenly over the zones, irrespective of the expected demand. This strategy leads, in comparison to the other two strategies, to the highest number of kilometres driven by SAV per served trip, as the SAV have longer access routes for reaching pick-up locations of passengers compared to relocation strategies taking into account future demand. This reduces the efficiency of the fleet in that regard. However, distributing idle vehicles evenly over zones leads to a more balanced usage of parking facilities.

The strategy Demand-Supply Balancing aims at reducing the deficit between future demand and future vehicle supply per zone, and thus combines the goals of the previous two strategies. The resulting KPI for this strategy are consequently also situated in most cases between those of the two previous relocation strategies, with the outcome of the Demand-Supply Balancing strategy being much closer to the one of the strategy Supply Anticipation than for Demand Anticipation. Two KPIs stand out in this regard, namely the empty driven mileage, and the passenger waiting times. This strategy leads to the highest value for VKT without passengers on board, which is caused in particular by the relocation of idle vehicles. As a consequence, this strategy also leads to the highest congestion levels in the network. At the same time, this strategy also leads to the shortest average passenger waiting times and the most equal distribution of the latter.

As shown in this study, the underlying principles of a vehicle relocation strategy impact the efficiency, externalities and equity of an on-demand service. It depends on the importance one attaches to these aspects, whether one declares one of these relocation strategies to be more beneficial than the others, since the results suggest that none of them outperforms the others in all regards. When discussing the introduction of SAV to a city, main stakeholders include the (potential) users of the transport service they offer, the operator of the service, the planning authority supervising the introduction of the new service to provide a certain level of service, and the citizens (potential non-users) in the area. From the perspective of a service operator, different relocation strategies can prove to be beneficial: Demand Anticipation allows to reduce average waiting times in high-demand areas and to reduce driven mileage, both contributing to increased service efficiency. For this reason, 
most current on-demand transport services operated by drivers acting as decision-making agents are dominated by this relocation strategy. When drivers are in direct competition for passengers with each other, they create a situation closer to a stochastic user equilibrium (SUE), which does not necessarily benefit the fleet as a whole. However, once the demand, and accordingly the fleet size, reaches a level that the operation of SAV causes local congestion, it can be beneficial for an operator to cap the number of idle vehicles in high-demand areas and swap to a relocation strategy which spreads out idle vehicles more, such as the strategy Supply Anticipation. Increased service efficiency is also beneficial for the users of the service in terms of reducing waiting times and in-vehicles times. For passengers requesting the service in zones with low demand, a relocation strategy distributing idle vehicles more evenly is particularly beneficial, as this reduces the waiting times that they might experience otherwise. The best results in this regard could be achieved with the strategy of Demand-Supply Balancing for the simulated case study.

When taking the perspective of a higher planning authority, for example on a municipal level, different objectives could be leading for selecting a relocation strategy. On-demand transport shows more fluctuation over time in its performance than scheduled transport, especially regarding waiting times and reliability of indicated waiting times. There is no clear consensus yet on how to benchmark average waiting times, maximum waiting times, reliability of indicated waiting times and updated waiting times for on-demand transport services. For the simulated scenarios, the service with the overall shortest trip times (waiting time and in-vehicle time combined) is achieved with the strategy Demand Anticipation. This strategy also leads to the lowest value for the total driven mileage, which can be interpreted as a proxy for energy consumption and emissions of pollutants and noise caused by this transport service. However, in regard to parking consumption, the case could be made for different strategies: Supply Anticipation and Demand-Supply Balancing lead to the much more equal occupation of parking facilities in spatial terms, which can reduce pressure on the parking facilities in popular areas like city centres, and also to a higher service provision equity. Another way to look at the bunching of idle vehicles in areas of high demand caused by the strategy Demand Anticipation could be to interpret this as a "polluter-pays" situation: if users of other modes are not affected by the parking consumption of SAV, e.g. because these park on reserved parking spots or private ground, and the local congestion the create SAV does not affect overall network flows, this could also be an acceptable solution from the perspective of a planning authority. Which relocation principle is more favourable for a city's management of scarce parking facilities depends on the local situation, and also in regard to which potential user groups might profit most from this. As neither the strategy of Demand Anticipation nor the one of Supply Anticipation clearly outperforms the other when taking into account the holistic set of KPI presented in this paper, a compromising strategy like the Demand-Supply Balancing strategy has the potential to provide the necessary attenuation of undesired effects.

\section{Study limitations and outlook}

The analysis of the relocation heuristics is based on the simulation of a case study. This setup does not allow to draw universal conclusions, and should be generalized or transferred to other contexts with caution. The main limitations of this study are linked to two input parameters: (1) the zonal division and (2) the behavioural model used to describe the users' response in the agent-based model. 
(1) The zonal division is expected to have an important impact on the working of vehicle relocation strategies based on demand and vehicle supply aggregated on a zonal level. For this study, the zones have been based on postal code areas which are commonly used in the Netherlands for defining parking regulations. However, depending on the goal of a relocation strategy, other criteria for the zonal divisions could be selected, e.g. a zonal division based on the current quality of scheduled public transport services. More research is required to determine the optimal (or good enough) number of zones, their size, and their defining principles in order to come to satisfying conclusions on zonal relocation strategies under parking constraints.

(2) In regard to the behavioural model used, the current state of research on mode choice behaviour in an era of SAV is not developed enough in order to confidently apply choice models to simulation models. In this paper, basic assumptions have been made about the specifications of the SAV service and on the perception of the different elements linked to a trip taken in an SAV. As for now, any simulation study featuring SAV can only be interpreted in light of the assumptions made for the underlying behavioural model.

Further limitations of this study are caused by other simplifications made for the simulation of the case study, such as that parking spots are dedicated for SAV and can be reserved upfront by the operator. Future research into the distribution of parking spots for SAV and the allocation of free parking spots will be an important aspect in the development of dynamic fleet operation paradigms. This is particularly important if more than one fleet of SAV compete with each other for parking space, or if they compete with individual drivers of (non-)automated vehicles. Furthermore, the performance of the different relocation strategies for idle vehicles should also be tested for other operational scenarios, most importantly for SAV operated as a pooled service. The operational differences for pooled services mainly impact the vehicle routing problem and vehicle dispatching problem, however, also the performance of vehicle relation strategies can change due to pooling. When operating SAV as a simultaneously shared service, instead of a sequentially shared one, vehicles likely turn idle less often, and they turn idle at different locations in the network. Which relocation strategy performs best in such a setting highly depends on local conditions and should be carefully tested for the different performance indicators presented in this study. The analysis in this study is based on one specific case study in order to retrace the impact of three simple vehicle relocation heuristics. Based on this set-up, future work will investigate further the interplay between the fleet size of SAV and the number of reserved parking facilities, and curbside management strategies will be concretized in order to test the impact of parking policies for SAV.

Acknowledgements The work of the first author is funded by the NWO TRAIL Graduate Programme.

\section{Compliance with ethical standards}

Conflict of interest On behalf of all authors, the corresponding author states that there is no conflict of interest.

Open Access This article is licensed under a Creative Commons Attribution 4.0 International License, which permits use, sharing, adaptation, distribution and reproduction in any medium or format, as long as you give appropriate credit to the original author(s) and the source, provide a link to the Creative Commons licence, and indicate if changes were made. The images or other third party material in this article are included in the article's Creative Commons licence, unless indicated otherwise in a credit line to the 
material. If material is not included in the article's Creative Commons licence and your intended use is not permitted by statutory regulation or exceeds the permitted use, you will need to obtain permission directly from the copyright holder. To view a copy of this licence, visit http://creativecommons.org/licenses/by/4.0/.

\section{References}

Alonso-mora, J., Samaranayake, S., Wallar, A., Frazzoli, E., Rus, D.: On-demand high-capacity ride-sharing via dynamic trip-vehicle assignment. PNAS 114, 462-467 (2017). https://doi.org/10.1073/pnas.16116 75114

Anderson, D.N.: "Not just a taxi"? For-profit ridesharing, driver strategies, and VMT. Transportation (Amst). 41, 1099-1117 (2014). https://doi.org/10.1007/s11116-014-9531-8

Anderson, J.M., Kalra, N., Stanley, K.D., Sorensen, P., Samaras, C., Oluwatola, O.A.: Autonomous Vehicle Technology_A Guide for Policymakers. Rand Corporation, Santa Monica (2014)

Arentze, T.A., Timmermans, H.J.P.: A learning-based transportation oriented simulation system. Transp. Res. Part B 38, 613-633 (2004). https://doi.org/10.1016/j.trb.2002.10.001

Ashkrof, P., Correia, G., Cats, O., van Arem, B.: Impact of automated vehicles on travel mode preference for different trip purposes and distances. Transp. Res. Rec. 2673, 607-616 (2019). https://doi. org/10.1177/0361198119841032

Azevedo, C.L., Marczuk, K., Raveau, S., Soh, H., Adnan, M., Basak, K., Loganathan, H., Deshmunkh, N., Lee, D.-H., Frazzoli, E., Ben-Akiva, M.: Microsimulation of demand and supply of autonomous mobility on demand. Transp. Res. Rec. J. Transp. Res. Board. 2564, 21-30 (2016). https://doi. org/10.3141/2564-03

Babicheva, T., Burghout, W., Andreasson, I., Fail, N.: The matching problem of empty vehicle redistribution in autonomous taxi systems. Proc. Comput. Sci. 130, 119-125 (2018). https://doi.org/10.1016/j.procs .2018.04.020

Bailey, A., Clark, T.D.: Taxi management and route control: a systems study and simulation experiment. In: Swain, J.J., Goldsman, D., Crain, R.C., Wilson, J.R. (eds.) Proceedings of the 1992 Winter Simulation Conference, pp. 1217-1223 (1992)

Balmer, M., Rieser, M.: MATSim-T: architecture and simulation times. In: Bazzan, A., Klügl, F. (eds.) Multi-Agent Systems for Traffic and Transportation Engineering, pp. 57-78. Information Science Reference (2009)

Ben-Dor, G., Ben-Elia, E., Benenson, I.: Determining an optimal fleet size for a reliable shared automated vehicle ride-sharing service. Proc. Comput. Sci. 151, 878-883 (2019). https://doi.org/10.1016/J. PROCS.2019.04.121

Ben-Elia, E., Benenson, I.: A spatially-explicit method for analyzing the equity of transit commuters' accessibility. Transp. Res. Part A Policy Pract. 120, 31-42 (2019). https://doi.org/10.1016/J. TRA.2018.11.017

Bischoff, J., Maciejewski, M.: Simulation of city-wide replacement of private cars with autonomous taxis in Berlin. Proc. Comput. Sci. 83, 237-244 (2016a). https://doi.org/10.1016/j.procs.2016.04.121

Bischoff, J., Maciejewski, M.: Autonomous Taxicabs in Berlin - A Spatiotemporal Analysis of Service Performance. Transp. Res. Proc. 19, 176-186 (2016b). https://doi.org/10.1016/j.trpro.2016.12.078

Börjesson, M., Eliasson, J., Franklin, J.P.: Valuations of travel time variability in scheduling versus meanvariance models. Transp. Res. Part B Methodol. 46, 855-873 (2012). https://doi.org/10.1016/j. trb.2012.02.004

Bösch, P.M., Becker, F., Becker, H., Axhausen, K.W.: Cost-based analysis of autonomous mobility services. Transp. Policy (2018). https://doi.org/10.1016/j.tranpol.2017.09.005

Campello-Vicente, H., Peral-Orts, R., Campillo-Davo, N., Velasco-Sanchez, E.: The effect of electric vehicles on urban noise maps. Appl. Acoust. 116, 59-64 (2017). https://doi.org/10.1016/J.APACO UST.2016.09.018

Cetin, T., Deakin, E.: Regulation of taxis and the rise of ridesharing. Transp. Policy 76, 149-158 (2019). https://doi.org/10.1016/J.TRANPOL.2017.09.002

Chen, T.D., Kockelman, K.M., Hanna, J.P.: Operations of a shared, autonomous, electric vehicle fleet: implications of vehicle \& charging infrastructure decisions. Transp. Res. Part A Policy Pract. 94, 243254 (2016). https://doi.org/10.1016/j.tra.2016.08.020

Dandl, F., Bogenberger, K.: Comparing free-floating carsharing data and simulations of autonomous electric taxis in Munich. IEEE Trans. Intell. Transp. Syst. 20, 2037-2047 (2019). https://doi.org/10.1109/ TITS.2018.2857208 
Dandl, F., Hyland, M., Bogenberger, K., Mahmassani, H.S.: Evaluating the impact of spatio-temporal demand forecast aggregation on the operational performance of shared autonomous mobility fleets. Transportation (Amst). 46, 1975-1996 (2019). https://doi.org/10.1007/s11116-019-10007-9

de Looff, E., Correia, G., van Cranenburgh, S., Snelder, M., van Arem, B.: Potential changes in value of travel time as a result of vehicle automation: a casestudy in the Netherlands. TRB 97th Annu. Meet. Compend. Pap. 31 (2018)

Fagnant, D.J., Kockelman, K.M.: The travel and environmental implications of shared autonomous vehicles, using agent-based model scenarios. Transp. Res. Part C Emerg. Technol. 40, 1-13 (2014). https://doi. org/10.1016/j.trc.2013.12.001

Fagnant, D.J., Kockelman, K.M., Bansal, P.: Operations of a shared autonomous vehicle fleet for the Austin, Texas market. Transp. Res. Rec. J. Transp. Res. Board. 4, 98-106 (2015). https://doi. org/10.3141/2536-12

Gemeente Amsterdam: Amsterdamse Thermometer van de Bereikbaarheid, Amsterdam (2016)

Gemeente Amsterdam: Kerncijfers Amsterdam 2017, Amsterdam, The Netherlands (2018)

Gini, C.: Variabilità e mutabilità, contributo allo studio delle distribuzioni e relazione statistiche. Stud. Econ. dell'Univ. di Cagliari. 3, 1-158 (1912)

Gurumurthy, K.M., Kockelman, K.M., Simoni, M.D.: Benefits and costs of ride-sharing in shared automated vehicles across Austin, Texas: opportunities for congestion pricing. Transp. Res. Rec. 2673, 548-556 (2019). https://doi.org/10.1177/0361198119850785

Hörl, S., Ruch, C., Becker, F., Frazzoli, E., Axhausen, K.W.: Fleet operational policies for automated mobility: a simulation assessment for Zurich. Transp. Res. Part C Emerg. Technol. 102, 20-31 (2019). https ://doi.org/10.1016/J.TRC.2019.02.020

Horni, A., Nagel, K., Axhausen, K.W.: The Multi-Agent Transport Simulation MATSim. Ubiquity Press, London (2016)

International Transport Forum: Urban Mobility System Upgrade: How shared self-driving cars could change city traffic, Paris (2015)

Kole, P.J., Löhr, A.J., Van Belleghem, F., Ragas, A.: Wear and tear of tyres: a stealthy source of microplastics in the environment. Int. J. Environ. Res. Public Health. 14, 1265 (2017). https://doi.org/10.3390/ ijerph14101265

Maciejewski, M.: Dynamic transport services. In: Horni, A., Nagel, K., Axhausen, K.W. (eds.) The MultiAgent Transport Simulation MATSim, pp. 145-152. Ubiquity Press, London (2016)

Maciejewski, M., Bischoff, J.: Large-scale microscopic simulation of taxi services. Proc. Comput. Sci. 52, 358-364 (2015). https://doi.org/10.1016/j.procs.2015.05.107

Maciejewski, M., Bischoff, J.: Congestion effects of autonomous taxi fleets. Transport 33, 971-980 (2018). https://doi.org/10.3846/16484142.2017.1347827

Maciejewski, M., Bischoff, J., Nagel, K.: An assignment-based approach to efficient real-time city-scale taxi dispatching. Intell. Syst. IEEE. 31, 68-77 (2016). https://doi.org/10.1109/MIS.2016.2

Ministry of Transport Public Works and Water Management: Cycling in the Netherlands. Den Haag, The Netherlands (2009)

Nagel, K., Kickhoefer, B., Horni, A., Charypar, D.: A closer look at scoring. In: Horni, A., Nagel, K., Axhausen, K.W. (eds.) The Multi-Agent Transport Simulation MATSim, pp. 23-34. Ubiquity Press, London (2016)

OpenStreetMap Contributors: OpenStreetMap. Planet dump retrieved from https://planet.osm.org (2018)

Sayarshad, H.R., Chow, J.Y.J.: Non-myopic relocation of idle mobility-on-demand vehicles as a dynamic location-allocation-queueing problem. Transp. Res. Part E Logist. Transp. Rev. 106, 60-77 (2017). https://doi.org/10.1016/j.tre.2017.08.003

Simoni, M.D., Kockelman, K.M., Gurumurthy, K.M., Bischoff, J.: Congestion pricing in a world of selfdriving vehicles: an analysis of different strategies in alternative future scenarios. Transp. Res. Part C Emerg. Technol. 98, 167-185 (2019). https://doi.org/10.1016/J.TRC.2018.11.002

van Engelen, M., Cats, O., Post, H., Aardal, K.: Enhancing flexible transport services with demand-anticipatory insertion heuristics. Transp. Res. Part E Logist. Transp. Rev. 110, 110-121 (2018). https://doi. org/10.1016/j.tre.2017.12.015

van Ommeren, K., Lelieveld, M., de Pater, M., Goedhart, W.: Maatschappelijke kosten en baten van de fiets., Amsterdam and The Hague, The Netherlands (2012)

Winter, K., Narayan, J.: Amsterdam Scenario MATSim. 4TU.Centre for Research Data. Dataset (2019). https://doi.org/10.4121/uuid:6108ed85-7b24-455e-bd95-89d84e6306fa

Winter, K., Cats, O., van Arem, B., Martens, K.: Impact of relocation strategies for a fleet of shared automated vehicles on service efficiency, effectiveness and externalities. In: 5th IEEE Int. Conf. Model. Technol. Intell. Transp. Syst. MT-ITS 2017—Proc, pp. 844-849 (2017). https://doi.org/10.1109/mtits .2017 .8005630 
Wright, S., Emele, C.D., Fukumoto, M., Velaga, N.R., Nelson, J.D.: The design, management and operation of flexible transport systems: comparison of experience between UK, Japan and India. Res. Transp. Econ. 48, 330-338 (2014). https://doi.org/10.1016/j.retrec.2014.09.060

Zhang, R., Pavone, M.: Control of robotic mobility-on-demand systems: a queueing-theoretical perspective. Int. J. Rob. Res. 35, 186-203 (2016). https://doi.org/10.1177/0278364915581863

Zhang, W., Guhathakurta, S., Fang, J., Zhang, G.: Exploring the impact of shared autonomous vehicles on urban parking demand: an agent-based simulation approach. Sustain. Cities Soc. 19, $34-45$ (2015). https://doi.org/10.1016/j.scs.2015.07.006

Zhang, R., Rossi, F., Pavone, M.: Model predictive control of autonomous mobility-on-demand systems. Int. Conf. Robot. Autom. (2016). https://doi.org/10.1109/icra.2016.7487272

Zheng, Z., Rasouli, S., Timmermans, H.: Modeling taxi driver anticipatory behavior. Comput. Environ. Urban Syst. 69, 133-141 (2018). https://doi.org/10.1016/j.compenvurbsys.2018.01.008

Publisher's Note Springer Nature remains neutral with regard to jurisdictional claims in published maps and institutional affiliations.

Konstanze Winter graduated from Delft University of Technology, Department Transport \& Planning, in 2015 (MSc) and in $2020(\mathrm{PhD})$. Her research interests lie in the planning and management of urban traffic, shared mobility and the development of new forms of public transport. She is curious about the functionality of cities and how in the long term a sustainable way of living in urban agglomerations can be achieved. She currently works as an adivisor for traffic- and transport models at Rijkswaterstaat, the executive agency of the Dutch Ministry of Infrastructure and Water Management. Recent Publication: Winter, K. (2020): Providing Public Transport by Self-Driving Vehicles: User Preferences, Fleet Operation, and Parking Management (Doctoral dissertation, Delft University of Technology, Delft, The Netherlands). Retrieved from https ://doi.org/10.4233/uuid:9f5b51e1-4877-4e60-a9d0-69fa37fa834f.

Oded Cats is Associate Professor of Passenger Transport Systems at Delft University of Technology. Dr. Cats co-directs the Smart Public Transport Lab at TU Delft, leading a research group that works closely with public transport authorities and operators. His research develops methods and models of multimodal metropolitan passenger transport systems by combining advancements from behavioural sciences, operations research and complex network theory. His research contributions focus on the development of dynamic transit assignment models, the optimisation of passenger service operations, network robustness analysis and real-time control methods. His research activities include the deployment of automated vehicles in passenger systems and their integration in the planning and operations of public transport services. He is the recipient of a personal European Research Council starting grant devoted to the network, operations, behavioral and mass effects related to mobility on demand (CriticalMaaS).

Karel Martens is Associate Professor and Chair of the Graduate Program in Urban and Regional Planning at the Faculty of Architecture and Town Planning, Technion - Israel Institute of Technology (Haifa, Israel), where he also heads the Fair Transport Lab. He holds a master degree in Spatial Planning (1991) and a PhD in Policy Sciences (2000), both from Radboud University, the Netherlands. His research interests include the nexus between transport and justice, transport and land use interaction, and parking dynamics and policies. He has authored numerous publications on parking policy as well as on equity assessment of transport systems and policies. His book Transport Justice: Designing Fair Transportation Systems has been described by colleagues as "ground-breaking", a "landmark" and "a revolution".

Bart van Arem was appointed full professor Transport Modelling at TU Delft in 2009. He was head of the department of Transport \& Planning at TU Delft from 2010-2017. Since 2011 he has served as director of the TU Delft Transport Institute. From 2003-2012 he was part-time full professor at the University of Twente. From 1991-2009, he worked at TNO. Bart van Arem has an MSc (1986) and PhD (1990) degree in Applied Mathematics at the University of Twente, the Netherlands. His research interests focus on the analyses and modelling the implications of intelligent vehicles. Such implications vary from human factors to traffic flow on roads and networks. The research has a strong modelling and simulation component based on empirics wherever possible using our instrumented vehicles and driving simulator. He is founding editor of the IEEE Open Journal of Intelligent Transportation Systems. 